\title{
Semigroups and one-way functions
}

\author{
J.C. Birget
}

28 Nov. 2015

To Stuart Margolis on his 60th birthday.

\begin{abstract}
We study the complexity classes P and NP through a semigroup fP ("polynomial-time functions"), consisting of all polynomially balanced polynomial-time computable partial functions. The semigroup $\mathrm{fP}$ is non-regular iff $\mathrm{P} \neq \mathrm{NP}$. The one-way functions considered here are based on worstcase complexity (they are not cryptographic); they are exactly the non-regular elements of fP. We prove various properties of $\mathrm{fP}$, e.g., that it is finitely generated. We define reductions with respect to which certain universal one-way functions are fP-complete.
\end{abstract}

Keywords: Semigroups, P vs. NP, regular semigroups.

Mathematics Subject Classification 2010: 20M05, 20M17, 68Q17, 68Q15

\section{Introduction}

The goal of this work is to study the complexity classes $\mathrm{P}$ and NP via functions, and via semigroups of functions, rather than just as sets of languages. This approach is intuitive (in particular, because of the immediate connection with certain one-way functions), and quickly leads to results. It is not clear whether this will contribute to a solution of the famous P-vs.-NP problem, but the semigroups considered here, as well as the "inversive reductions" and the accompanying completeness results for one-way functions, are interesting in their own right.

The starting point is a certain kind of one-way functions, and the well-known fact that one-way functions of this kind exist iff $P \neq N P$.

First, some notation: We fix an alphabet $A$, which will be $\{0,1\}$ unless another alphabet is explicitly mentioned. The set of all strings over $A$, including the empty string, is denoted by $A^{*}$. For a partial function $f$, the domain (i.e., the inputs $x$ for which $f(x)$ is defined) is denoted by $\operatorname{Dom}(f)$. The image set of $f$ is denoted by $\operatorname{Im}(f)$ or by $f\left(A^{*}\right)$ or $f(\operatorname{Dom}(f))$. As a rule we will use partial functions, even when the word "partial" is omitted; we say total function for functions whose domain is $A^{*}$. As usual, P and NP are the class of languages accepted by deterministic, respectively nondeterministic, polynomial-time Turing machines [11, 22].

Definition scheme: A function $f: A^{*} \rightarrow A^{*}$ is called "one-way" iff from $x$ and a description of $f$ it is "easy" to compute $f(x)$, but from $f$ and $y \in \operatorname{Im}(f)$ it is "difficult" to find any $x \in A^{*}$ such that $f(x)=y$.

This is an old idea going back at least to W.S. Jevons in 1873, who also compared the difficulties of multiplication and factorization of integers (as pointed out in [21]). The concept became well-known after the work of Diffie and Hellman [10. Levin's paper [19] discusses some deeper connections of oneway functions. The definition scheme can be turned into precise definitions, in many (non-equivalent) ways, by defining "easy" and "difficult" (and, if needed, "description" of $f$ ). 
Definition 1.1 A partial function $f: A^{*} \rightarrow A^{*}$ is polynomially balanced iff there exists polynomials $p, q$ such that for all $x \in \operatorname{Dom}(f): \quad|f(x)| \leq p(|x|)$ and $|x| \leq q(|f(x)|)$.

We call the polynomial $q$ above an input balance function of $f$. The word "honest" is often used in the literature for polynomially balanced.

We introduce the following set of "easy" functions.

Definition 1.2 (the semigroup fP). fP is the set of partial functions $f: A^{*} \rightarrow A^{*}$ that are polynomially balanced, and such that $x \in \operatorname{Dom}(f) \longmapsto f(x)$ is computable by a deterministic polynomial-time Turing machine. (It follows from the second condition that $\operatorname{Dom}(f)$ is in $\mathrm{P}$.)

As a rule, a machine that computes a partial function $f$ can always also be used as an acceptor of $\operatorname{Dom}(f)$.

When $A$ is an arbitrary alphabet (as opposed to $\{0,1\}$ ) we write $\mathrm{fP}_{A}$ or $\mathrm{fP}_{|A|}$. The complexity class $\mathrm{fP}$ is different from the complexity class FP, considered in the literature [22]; FP is a set of relations (viewed as search problems) whereas $\mathrm{fP}$ is a set of partial functions. It is easy to see that $\mathrm{fP}_{|A|}$ is closed under composition, so it is a semigroup.

Definition 1.3 (worst-case deterministic one-way function). A partial function $f: A^{*} \rightarrow A^{*}$ is one-way iff $f \in \mathrm{fP}$, but there exists no deterministic polynomial-time algorithm which, on every input $y \in \operatorname{Im}(f)$, outputs some $x \in A^{*}$ such that $f(x)=y$. There is no requirement when $y \notin \operatorname{Im}(f)$.

This kind of one-way functions is defined in terms of worst-case complexity, hence it is not "cryptographic". However, it is relevant to the P-vs.-NP problem because of the following fact (see e.g., [15] p. 33 for a proof and history).

Proposition 1.4 (folklore). One-way functions (in the worst-case sense) exist iff $\mathrm{P} \neq \mathrm{NP}$.

The concept of an inverse is central to one-way functions. The following lemma is straightforward to prove.

Notation: For a partial function $f$ and a set $S$, the restriction of $f$ to $S$ is denoted by $\left.f\right|_{S}$; for the restriction of the identity map to $S$ we simply write $\operatorname{id}_{S}$.

Lemma 1.5 (concept of inverse).

For partial functions $f, f^{\prime}: A^{*} \rightarrow A^{*}$ the following are equivalent.

- For all $y \in \operatorname{Im}(f), f^{\prime}(y)$ is defined and $f\left(f^{\prime}(y)\right)=y$.

- $\left.f \circ f^{\prime}\right|_{\operatorname{Im}(f)}=\mathrm{id}_{\operatorname{Im}(f)}$.

- $f \circ f^{\prime} \circ f=f$.

These properties imply $\operatorname{Im}(f) \subseteq \operatorname{Dom}\left(f^{\prime}\right)$.

Definition 1.6 A function $f^{\prime}$ such that $f \circ f^{\prime} \circ f=f$ is called an inverse of $f$.

The following recipe gives more intuition about inverses.

Pseudo-algorithm: How any inverse $f^{\prime}$ of a given $f$ is made.

(1) Choose $\operatorname{Dom}\left(f^{\prime}\right)$ such that $\operatorname{Im}(f) \subseteq \operatorname{Dom}\left(f^{\prime}\right)$.

(2) For every $y \in \operatorname{Im}(f)$, choose $f^{\prime}(y)$ to be any $x \in f^{-1}(y)$.

(3) For every $y \in \operatorname{Dom}\left(f^{\prime}\right)-\operatorname{Im}(f)$, choose $f^{\prime}(y)$ arbitrarily in $A^{*}$.

Remark. When $f^{\prime}$ is an inverse of $f$, the restriction $\left.f^{\prime}\right|_{\operatorname{Im}(f)}: y \in \operatorname{Im}(f) \mapsto f^{\prime}(y)$ is the choice function corresponding to $f^{\prime}$. For set theory in general, the existence of choice functions (and the existence of inverses) for every partial function is equivalent to the Axiom of Choice. The existence of 
one-way functions in our sense amounts to the non-existence of certain inverses; the existence of oneway functions is thus equivalent to the non-validity of the Axiom of Choice in the (highly restricted) context of deterministic polynomial time-complexity.

From the definition of polynomially balanced we can see now: If $f$ is polynomially balanced then so is every choice function corresponding to any inverse of $f$.

Definition 1.7 Let $S$ be a semigroup. An element $x \in S$ is called regular iff there exists $x^{\prime} \in S$ such that $x x^{\prime} x=x$. In that case, $x^{\prime}$ is called an inverse of $x$. A semigroup $S$ is called regular iff every element of $S$ is regular.

Let $S$ be a monoid with identity $\mathbf{1}$. Then $x^{\prime}$ is a left- (or right-) inverse of $x$ iff $x^{\prime} x=\mathbf{1}$ (respectively $x x^{\prime}=1$ ). If $x^{\prime} x=x x^{\prime}=\mathbf{1}$ then $x^{\prime}$ is a two-sided inverse or group-inverse. (See [9, 13].)

The following summarizes what we have seen, and gives the initial motivation for studying the class NP via certain functions and semigroups.

Proposition 1.8 The monoid $\mathrm{fP}$ is regular iff one-way functions do not exist (iff $\mathrm{P}=\mathrm{NP}$ ).

Some properties of the image set of functions in fP:

\section{Proposition 1.9 .}

(1) For every $f \in \mathrm{fP}, \quad \operatorname{Im}(f) \in \mathrm{NP}$.

(2) If $f \in \mathrm{fP}$ and $f$ is regular then $\operatorname{Im}(f) \in \mathrm{P}$.

(3) For every language $L \in \mathrm{NP}$ there exists $f_{L} \in \mathrm{fP}$ such that $L=\operatorname{Im}\left(f_{L}\right)$.

Moreover, the set of functions $\left\{f_{L} \in \mathrm{fP}: L \in \mathrm{NP}\right\}$ can be chosen so that $f_{L}$ is regular iff $L \in \mathrm{P}$. The map $L \in \mathrm{NP} \mapsto f_{L} \in \mathrm{fP}$ is an embedding of NP (as a set) into $\mathrm{PP}$, such that $\mathrm{P}$ (and only $\mathrm{P}$ ) is mapped into the regular elements of $\mathrm{fP}$.

Proof. (1) is obvious (polynomial balance is needed).

(2) Let $f^{\prime} \in \mathrm{fP}$ be an inverse of $f$. If $y \in \operatorname{Im}(f)$ then $f f^{\prime}(y)=y$. If $y \notin \operatorname{Im}(f)$, then either $f^{\prime}(y)$ is not defined, or $f f^{\prime}(y) \in \operatorname{Im}(f)$, hence $f f^{\prime}(y) \neq y$. Thus, $y \in \operatorname{Im}(f)$ iff $f f^{\prime}(y)=y$. When $f, f^{\prime} \in \mathrm{fP}$ then on input $y$ the properties $f f^{\prime}(y)=y, y \notin \operatorname{Dom}\left(f^{\prime}\right), f f^{\prime}(y) \neq y$, can be decided deterministically in polynomial time.

(3) Let $M$ be a nondeterministic Turing machine accepting $L$, such that all computations of $M$ are polynomially bounded, and do not halt before the whole input has been read. We can assume that $M$ has binary nondeterminism, i.e., in each transition it has at most two nondeterministic choices. Define

$f_{L}(x, s)=x \quad$ iff $\quad M$, with choice sequence $s$, accepts $x$ $f_{L}(x, s)$ is undefined otherwise.

Choice sequences are also called guessing sequences, or advice sequences. Then $L=\operatorname{Im}\left(f_{L}\right)$ and $f_{L} \in \mathrm{fP}$; balancing comes from the fact that $s$ has polynomially bounded length.

We saw that if $f_{L}$ is regular then $\operatorname{Im}\left(f_{L}\right)=L \in \mathrm{P}$. Moreover, if $L \in \mathrm{P}$ then the Turing machine $M$ can be chosen to be deterministic, and the choice sequence $s$ is the all-0 word; so in that case, $f_{L}$ is not one-way.

Corollary 1.10 The image transformation Im: $f \mapsto \operatorname{Im}(f)$ maps fP onto NP, and maps the set of regular elements of $\mathrm{fP}$ onto $\mathrm{P}$. Moreover, NP is embedded into $\mathrm{fP}$ (by the transformation $L \mapsto f_{L}$ above), and NP is a retract of $\mathrm{fP}$ (by the transformations $L \mapsto f_{L}$ and $\mathrm{Im}$ ).

The following suggests that $\operatorname{Im}(f) \in \mathrm{P}$ is not equivalent to the regularity of $f$.

Theorem 1.11 If $\Pi_{2}^{\mathrm{P}} \neq \Sigma_{2}^{\mathrm{P}}$ then there exist surjective one-way functions. (Proved in [7].)

In the next sections we show various properties of fP and of closely related semigroups. The fact that these semigroups have interesting properties, and that the proofs are not difficult, gives a second motivation for studying these semigroups. 


\section{The Green relations of polynomial-time function semigroups}

We give a few properties of the Green relations $\leq_{\mathcal{R}}, \leq_{\mathcal{L}}, \leq_{\mathcal{J}}$, $\equiv_{\mathcal{D}}$ (see [9, 13]). First some notation.

Let $F: X \rightarrow Y$ be a partial function; then $F^{-1}: Y \rightarrow X$ denotes the inverse relation of $F$, i.e., for all $(x, y) \in X \times Y: \quad x \in F^{-1}(y)$ iff $F(x)=y$. By $\bmod F$ we denote the partition on $\operatorname{Dom}(F)$, defined by $x_{1} \bmod F x_{2}$ iff $F\left(x_{1}\right)=F\left(x_{2}\right)$. The set of blocks (equivalence classes) of $\bmod F$ is $\left\{F^{-1} F(x): x \in \operatorname{Dom}(F)\right\}$. For two partial functions $F, C: X \rightarrow Y$ we write $\bmod C \leq \bmod F$ (the partition of $C$ is coarser than the partition of $F$, or the partition of $F$ refines the partition of $C$ ) iff $\operatorname{Dom}(C) \subseteq \operatorname{Dom}(F)$ and $F^{-1} F(x) \subseteq C^{-1} C(x)$ for all $x \in \operatorname{Dom}(C)$; equivalently, every mod $C$-class is a union of $\bmod F$-classes.

Proposition 2.1 (regular $\mathcal{L}$ - and $\mathcal{R}$-orders). If $f, r \in \mathrm{fP}$ and $r$ is regular with an inverse $r^{\prime} \in \mathrm{fP}$ then:

- $f \leq_{\mathcal{R}} r \quad$ iff $\quad f=r r^{\prime} f \quad$ iff $\operatorname{Im}(f) \subseteq \operatorname{Im}(r)$.

- $f \leq_{\mathcal{L}} r$ iff $f=f r^{\prime} r \quad$ iff $\bmod f \leq \bmod r$.

Proof. [R -order]: $f \leq_{\mathcal{R}} r$ iff for some $u \in \mathrm{fP}: f=r u$. Then $f=r r^{\prime} r u=r r^{\prime} f$. Also, it is straightforward that $f=r u$ implies $\operatorname{Im}(f) \subseteq \operatorname{Im}(r)$.

Conversely, if $\operatorname{Im}(f) \subseteq \operatorname{Im}(r)$ then $\mathrm{id}_{\operatorname{Im}(f)}=\mathrm{id}_{\operatorname{Im}(r)} \circ \mathrm{id}_{\operatorname{Im}(f)}=\left.r \circ r^{\prime}\right|_{\operatorname{Im}(r)} \circ \mathrm{id}_{\operatorname{Im}(f)}$. Hence, $f=\mathrm{id}_{\operatorname{Im}(f)} \circ f=\left.r \circ r^{\prime}\right|_{\operatorname{Im}(r)} \circ \mathrm{id}_{\operatorname{Im}(f)} \circ f=\left.r \circ r^{\prime}\right|_{\operatorname{Im}(r)} \circ f \leq_{\mathcal{R}} r$.

$\left[\mathcal{L}\right.$-order]: $f \leq_{\mathcal{L}} r$ iff for some $v \in \mathrm{fP}: f=v r$. Then $f=v r r^{\prime} r=f r^{\prime} r$. And it is straightforward that $f=v r$ implies $\bmod f \leq \bmod r$.

Conversely, if $\bmod f \leq \bmod r$ then for all $x \in \operatorname{Dom}(f), r^{-1} r(x) \subseteq f^{-1} f(x)$. And for every $x \in \operatorname{Dom}(f), \quad\{f(x)\}=f \circ f^{-1} \circ f(x)$. Moreover, $f \circ r^{-1} \circ r(x) \subseteq f \circ f^{-1} \circ f(x)=\{f(x)\}$, and since $r^{-1} \circ r(x) \neq \varnothing$, it follows that $f \circ r^{-1} \circ r(x)=\{f(x)\}$. So, $f=f \circ r^{-1} \circ r$. Moreover, $f \circ r^{\prime} \circ r(x) \in f \circ r^{-1} \circ r(x)=\{f(x)\}$, hence $f \circ r^{\prime} \circ r(x)=f(x)$. Hence, $f=f r^{\prime} r \leq_{\mathcal{L}} r$.

The $\mathcal{D}$-relation between elements of fP with infinite image sets seems difficult, even in the case of regular elements. A first question (inspired from the Thompson-Higman monoids [3]): Are all regular elements of fP with infinite image in the same $\mathcal{D}$-class, i.e., the $\mathcal{D}$-class of $\mathrm{id}_{A^{*}}$ ?

Proposition 2.2 Let $f \in \mathrm{fP}$ be regular. Then $f \equiv_{\mathcal{D}} \mathrm{id}_{A^{*}}$ iff there exists $g \in \mathrm{fP}$ such that $g$ is injective, total, and regular, and such that $\operatorname{Im}(f)=\operatorname{Im}(g)$.

Proof. Assume $f \equiv_{\mathcal{D}} \mathrm{id}_{A^{*}}$. By Prop. 2.1, $f \equiv_{\mathcal{R}} \mathrm{id}_{L}$, where $L=\operatorname{Im}(f)$. And $\mathrm{id}_{A^{*}} \equiv_{\mathcal{D}} \mathrm{id}_{L}$ iff there exists $g \in \mathrm{fP}$ such that $\mathrm{id}_{A^{*}} \equiv_{\mathcal{L}} g \equiv_{\mathcal{R}} \mathrm{id}_{L}$. Hence, $\mathrm{id}_{A^{*}}=g^{\prime} g$ for some $g^{\prime} \in \mathrm{fP}$; this equality implies that $g$ is total and injective. The existence of $g^{\prime} \in \mathrm{fP}$ implies that $g$ is regular. Since $g \equiv_{\mathcal{R}} \mathrm{id}_{L}$, $\operatorname{Im}(g)=L$. Hence, $g$ has the required properties.

To prove the converse we will use the following:

Claim. For every $g \in \mathrm{fP}$ we have: $g$ is injective, total, and regular iff $\left(\exists g^{\prime} \in \mathrm{fP}\right) g^{\prime} g=\mathrm{id}_{A^{*}}$.

Proof of the Claim. The right-to-left implication is straightforward. In the other direction, if $g$ is regular then there exists $g^{\prime} \in \mathrm{fP}$ such that $g g^{\prime} g=g$. And if $g$ is total and injective, there exists a partial function $h$ such that $h g=\mathrm{id}_{A^{*}}$. Now $g g^{\prime} g=g$ implies $h g g^{\prime} g=h g$, hence by using $h g=\mathrm{id}_{A^{*}}$ we obtain: $g^{\prime} g=\mathrm{id}_{A^{*}}$. This proves the Claim.

For the converse of the Proposition, assume there exists $g \in \mathrm{fP}$ with the required properties. If such a $g$ exists, then $f \equiv_{\mathcal{R}} g$, by Prop. 2.1. Moreover, $g \equiv_{\mathcal{L}} \mathrm{id}_{A^{*}}$; this follows from $g^{\prime} g=\mathrm{id}_{A^{*}}$, which holds by the Claim. Hence $f \equiv_{\mathcal{R}} g \equiv_{\mathcal{L}}$ id $_{A^{*}}$.

However, it is an open problem whether every infinite language $L$ in $\mathrm{P}$ is the image of an injective, total, polynomial-time computable function $g$ (and whether $g$ can be taken to be regular or one-way). 
Also, not much is known about which infinite languages in $\mathrm{P}$ can be mapped onto each other by maps in $\mathrm{fP}$.

When $\operatorname{Im}(f)$ is a right ideal, more can be said. By definition, a right ideal of $A^{*}$ is a subset $R \subseteq A^{*}$ such that $R A^{*}=R$ (i.e., $R$ is closed under right-concatenation by any string). Equivalently, a right ideal is a set of the form $R=L A^{*}$, for any set $L \subseteq A^{*}$; in that case we also say that $L$ generates $R$ as a right ideal. A prefix code in $A^{*}$ is a set $P \subseteq A^{*}$ such that no word in $P$ is a prefix of another word in $P$. It is not hard to prove that for any right ideal $R$ there exists a unique prefix code $P_{R}$ such that $R=P_{R} A^{*}$; in other words, $P_{R}$ is the minimum generating set of $R$, as a right ideal.

A right ideal morphism is a partial function $h: A^{*} \rightarrow A^{*}$ such that for all $x \in \operatorname{Dom}(h)$ and all $w \in A^{*}: \quad h(x w)=h(x) w$. One proves easily that then $\operatorname{Dom}(h)$ and $\operatorname{Im}(h)$ are right ideals.

We also consider $A^{\omega}$ (the $\omega$-sequences over $A$, see e.g. [23]). For a set $L \subseteq A^{*}$ we define ends $(L)$ to consist of all elements of $A^{\omega}$ that have a prefix in $L$. The Cantor space topology on $A^{\omega}$ uses the sets of the form ends $(L)$ (for $L \subseteq A^{*}$ ) as its open sets; here we can assume without loss of generality that $L$ is a prefix code or a right ideal of $A^{*}$.

Lemma 2.3 If a right ideal $R \subseteq A^{*}$ belongs to $\mathrm{P}$ then the corresponding prefix code $P$ (such that $R=P A^{*}$ ) also belongs to $\mathrm{P}$. Conversely, if $L$ is in $\mathrm{P}$ then $L A^{*}$ is in $\mathrm{P}$.

Proof. The first statement follows immediately from the fact that $x \in P$ iff $x \in R$ and every strict prefix of $x$ does not belong to $R$. The converse is straightforward.

Notation. Below, $\overline{P A^{*}}$ denotes $A^{*}-P A^{*}$ (complement).

Proposition 2.4 Let $P \subseteq A^{*}$ be a prefix code that belongs to $\mathrm{P}$, and let $p_{0} \in P$. Then all regular elements $r \in \mathrm{fP}$ whose image is $\operatorname{Im}(r)=L_{P}=\left(P-\left\{p_{0}\right\}\right) A^{*} \cup p_{0}\left(p_{0} A^{*} \cup \overline{P A^{*}}\right)$ are in the $\mathcal{D}$-class of $\mathrm{id}_{A^{*}}$. We can view $L_{P}$ as an "approximation" of the right ideal $P A^{*}$ since

$$
\left(P-\left\{p_{0}\right\}\right) A^{*} \subset L_{P} \subset P A^{*} .
$$

Proof. Let $L=L_{P}=\operatorname{Im}(r)$. By Prop. 2.1, $r \equiv_{\mathcal{R}} \mathrm{id}_{L}$, so it suffices to prove that $\mathrm{id}_{L} \equiv_{\mathcal{D}} \mathrm{id}_{A^{*}}$. We define $\pi, \pi^{\prime} \in \mathrm{fP}$ by

$$
\begin{aligned}
& \pi(x)= \begin{cases}x & \text { if } x \in\left(P-\left\{p_{0}\right\}\right) A^{*}, \\
p_{0} x & \text { otherwise (i.e., if } \left.x \in p_{0} A^{*} \text { or } x \notin P A^{*}\right) ;\end{cases} \\
& \pi^{\prime}(x)= \begin{cases}x & \text { if } x \in\left(P-\left\{p_{0}\right\}\right) A^{*}, \\
z & \text { if } x \in p_{0} A^{*} \text { with } x=p_{0} z, \\
\text { undefined } & \text { otherwise (i.e., when } \left.x \notin P A^{*}\right) .\end{cases}
\end{aligned}
$$

Then $\pi$ is a total and injective function, and $\operatorname{Im}(\pi)=L$. Hence, $\pi \equiv_{\mathcal{R}} \mathrm{id}_{L}$. Moreover, $\pi^{\prime} \circ \pi=\mathrm{id}_{A^{*}}$, as is easily verified, hence $\pi \equiv_{\mathcal{L}} \mathrm{id}_{A^{*}}$. In summary, $r \equiv_{\mathcal{R}} \mathrm{id}_{L} \equiv_{\mathcal{R}} \pi \equiv_{\mathcal{L}} \mathrm{id}_{A^{*}}$.

Functions that have right ideals as domain and image are of great interest, because of the remarkable properties of the Thompson-Higman groups and monoids [20, 25, 24, 17, 8, 6, 5] and [2, 3, 4]. Prop. 2.4 gives an additional motivation for looking at the special role of right ideals. This motivates the following.

Definition 2.5 $\mathcal{R} \mathcal{M}_{|A|}^{\mathrm{P}}=\left\{f \in \mathrm{fP}: f\right.$ is a right ideal morphism of $\left.A^{*}\right\}$.

When $f$ is a right ideal morphism, $\operatorname{Dom}(f)$ and $\operatorname{Im}(f)$ are right ideals. $\mathcal{R} \mathcal{M}_{|A|}^{\mathrm{P}}$ is closed under composition, and $\mathcal{R} \mathcal{M}_{2}^{\mathrm{P}}$ is a submonoid of $\mathrm{fP}$.

An interesting submonoid of $\mathcal{R} \mathcal{M}_{|A|}^{\mathrm{P}}$ is $\mathcal{R} \mathcal{M}_{|A|}^{\text {fin }}$, consisting of all those $f \in \mathcal{R} \mathcal{M}_{|A|}^{\mathrm{P}}$ for which $\operatorname{Dom}(f)$ (and hence also $\operatorname{Im}(f)$ ) is a finitely generated right ideal. The monoid $\mathcal{R} \mathcal{M}_{|A|}^{\text {fin }}$ is used to define the Thompson-Higman monoid $M_{|A|, 1}$ in [3]. 
Proposition 2.6 If an element $f \in \mathcal{R} \mathcal{M}_{2}^{\mathrm{P}}$ has an inverse in $\mathrm{fP}$ then $f$ also has an inverse in $\mathcal{R} \mathcal{M}_{2}^{\mathrm{P}}$.

Proof. Let $f_{0}^{\prime} \in \mathrm{fP}$ be an inverse of $f$; we want to construct an inverse $f^{\prime}$ of $f$ that belongs to $\mathcal{R} \mathcal{M}_{2}^{\mathrm{P}}$. Since $f$ is regular in $\mathrm{fP}$, we know from Prop. 1.9 that $\operatorname{Im}(f)$ is in P. Hence we can restrict $f_{0}^{\prime}$ to $\operatorname{Im}(f)$, i.e., $\operatorname{Dom}\left(f_{0}^{\prime}\right)=\operatorname{Im}(f)$. We proceed to define $f^{\prime}(y)$ for $y \in \operatorname{Im}(f)$.

First, we compute the shortest prefix $p$ of $y$ that satisfies $p \in \operatorname{Dom}\left(f_{0}^{\prime}\right)=\operatorname{Im}(f)$. Since $\operatorname{Im}(f) \in \mathrm{P}$, this can be done in polynomial time. Now, $y=p z$ for some string $z$.

Second, we define $f^{\prime}(y)=f_{0}^{\prime}(p) z$, where $p$ and $z$ are as above. Thus, $f^{\prime}$ is a right-ideal morphism.

Let us verify that $f^{\prime}$ has the claimed properties. Clearly, $f^{\prime}$ is polynomial-time computable, and polynomially balanced (the latter following from the fact that $f^{\prime}$ is an inverse of $f$, which we prove next). To prove that $f^{\prime}$ is an inverse of $f$, let $x \in \operatorname{Dom}(f)$. Then $f\left(f^{\prime}(f(x))\right)=f\left(f^{\prime}(p z)\right)$, where $y=f(x)=p z$, and $p$ is the shortest prefix of $y$ such that $p \in \operatorname{Im}(f)$. Then, $f^{\prime}(p z)=f_{0}^{\prime}(p) z$, by the definition of $f^{\prime}$. Then, since $f$ is a right-ideal morphism, $f\left(f_{0}^{\prime}(p) z\right)=f\left(f_{0}^{\prime}(p)\right) z=p z$ (the latter since $f_{0}^{\prime}$ is an inverse of $f$, and since $\left.p \in \operatorname{Im}(f)\right)$. Hence, $\left.f f^{\prime}\right|_{\operatorname{Im}(f)}=\mathrm{id}_{\operatorname{Im}(f)}$. Thus, by Prop. 1.5, $f^{\prime}$ is an inverse of $f$.

Remark and notation: For $f \in \mathcal{R} \mathcal{M}_{|A|}^{\mathrm{P}}$ we saw that $\operatorname{Dom}(f)$ and $\operatorname{Im}(f)$ are right ideals. Let $\operatorname{dom} C(f)$, called the domain code, be the prefix code that generates $\operatorname{Dom}(f)$ as a right ideal. Similarly, let $\operatorname{imC}(f)$, called the image code, be the prefix code that generates $\operatorname{Im}(f)$.

In general, $\operatorname{imC}(f) \subseteq f(\operatorname{dom} C(f))$, and it can happen that $\operatorname{imC}(f) \neq f(\operatorname{dom} C(f))$. However the last paragraph of proof of Prop. 2.6 shows that in any case: If $f \in \mathcal{R} \mathcal{M}_{|A|}^{\mathrm{P}}$ is regular then $f$ has an inverse $f^{\prime} \in \mathcal{R} \mathcal{M}_{|A|}^{\mathrm{P}}$ such that $\operatorname{dom} \mathrm{C}\left(f^{\prime}\right)=\operatorname{imC}(f)$.

Notation: For two words $u, v \in A^{*},(v \leftarrow u)$ denotes the right ideal morphism $u x \mapsto v x$ (for all $\left.x \in A^{*}\right)$. In particular, $(\varepsilon \leftarrow \varepsilon)=\mathrm{id}_{A^{*}}$, where $\varepsilon$ denotes the empty word. The morphism $(v \leftarrow u)$ is length-balanced because $|u|,|v|$ are constants for a given morphism.

Proposition 2.7 For every alphabet $A$, the monoid $\mathcal{R} \mathcal{M}_{|A|}^{\mathrm{P}}$ is $\mathcal{J}^{0}$-simple (i.e., the only ideals are $\{0\}$ and $\mathcal{R} \mathcal{M}_{|A|}^{\mathrm{P}}$ itself $)$.

Proof. For any $f \in \mathcal{R} \mathcal{M}_{|A|}^{\mathrm{P}}$ that is not the empty map, there exist words $x_{0}, y_{0}$ such that $f\left(x_{0}\right)=y_{0}$. Then $(\varepsilon \leftarrow \varepsilon)=\left(\varepsilon \leftarrow y_{0}\right) \circ f \circ\left(x_{0} \leftarrow \varepsilon\right)$. Hence, $\mathrm{id}_{A^{*}} \leq \mathcal{J} f$ for every non-empty element $f \in \mathcal{R} \mathcal{M}_{|A|}^{\mathrm{P}}$.

Proposition 2.8 fP is not $\mathcal{J}^{0}$-simple, and it has regular elements in different non-0 $\mathcal{J}$-classes.

Proof. The map $\ell: x \in\{0,1\}^{*} \longmapsto 0^{|x|}$ is in $\mathrm{fP}$ and it is an idempotent.

Moreover, $\ell \not \equiv \mathcal{J}$ id $_{A^{*}}$. Indeed, if there exist functions $\beta, \alpha$ such that for all $x \in A^{*}, x=\beta \ell \alpha(x)=$ $\beta\left(0^{|\alpha(x)|}\right)$, then $|\alpha(x)|$ is different for every $x \in A^{*}$. But then $\alpha$ is not polynomially balanced, since $|\alpha(x)|$ would have to range over $|A|^{|x|}$ values.

Corollary 2.9 fP and $\mathcal{R} \mathcal{M}_{|A|}^{\mathrm{P}}$ are not isomorphic.

As a consequence of Prop. 2.4 we have:

Corollary 2.10 Every regular element $r \in \mathcal{R} \mathcal{M}_{2}^{\mathrm{P}}$ is "close" to an element of fP belonging to the $\mathcal{D}$-class of $\mathrm{id}_{A^{*}}$. Here, $h_{p_{0}} \in \mathrm{fP}$ is called "close" to $r$ iff $\operatorname{Im}(r)=P A^{*}$ for a prefix code $P$, and there exists $p_{0} \in P$ such that:

- $\left(P-\left\{p_{0}\right\}\right) A^{*} \subseteq \operatorname{Im}\left(h_{p_{0}}\right) \subseteq P A^{*}$, and

- $h_{p_{0}}(x)=r(x)$ whenever $r(x) \in \operatorname{Im}\left(h_{p_{0}}\right)$. 
Proof. Let $P=\operatorname{dom} C(r)$, so $P A^{*}=\operatorname{Im}(r)$. For every $p_{0} \in P, r$ is close to $\mathrm{id}_{L_{P}} \circ r$, whose image set is $L_{P}=\left(P-\left\{p_{0}\right\}\right) A^{*} \cup p_{0}\left(p_{0} A^{*} \cup \overline{P A^{*}}\right)$, hence $\left(P-\left\{p_{0}\right\}\right) A^{*} \subset L_{P} \subset P A^{*}$. And id $L_{P} \circ r \equiv_{\mathcal{R}} \mathrm{id}_{L_{P}}$ since $L_{P} \subset P A^{*}$.

Recall the notation $(v \leftarrow u)$ given just before Prop. 2.7.

Lemma 2.11 In $\mathcal{R} \mathcal{M}_{2}^{\mathrm{P}}$, the $\mathcal{L}$-class of $\operatorname{id}_{A^{*}}$ is $\left\{(v \leftarrow \varepsilon) \in \mathcal{R} \mathcal{M}_{2}^{\mathrm{P}}: v \in A^{*}\right\}$. This is the set of elements of $\mathcal{R} \mathcal{M}_{2}^{\mathrm{P}}$ that are injective and total (i.e., defined for all $x \in A^{*}$ ).

The $\mathcal{R}$-class of $\operatorname{id}_{A^{*}}$ is $\left\{f \in \mathcal{R M}_{2}^{\mathrm{P}}: \varepsilon \in \operatorname{Im}(f)\right\}$. This is the set of elements of $\mathcal{R} \mathcal{M}_{2}^{\mathrm{P}}$ that are surjective (i.e., map onto $A^{*}$ ).

Proof. If $f \equiv_{\mathcal{L}} \operatorname{id}_{A^{*}}$ then $\varepsilon \in \operatorname{Dom}(f)=A^{*}$, so there is $v \in A^{*}$ such that $v=f(\varepsilon)$. Then $f(x)=v x$ for all $x \in A^{*}$. Conversely, if $f(x)=v x$ for all $x \in A^{*}$ then $(\varepsilon \leftarrow v) \circ f=\mathrm{id}_{A^{*}}$.

If $f \equiv_{\mathcal{R}} \mathrm{id}_{A^{*}}$ then $\operatorname{Im}(f)=A^{*}$. So $\varepsilon \in \operatorname{Im}(f)$. Conversely, if $f$ satisfies $\varepsilon \in \operatorname{Im}(f)$, i.e., $\varepsilon=f\left(x_{0}\right)$ for some $x_{0} \in A^{*}$, then $f \circ\left(x_{0} \leftarrow \varepsilon\right)=(\varepsilon \leftarrow \varepsilon)=\mathrm{id}_{A^{*}}$.

Lemma 2.11 shows that the $\mathcal{L}$-class of id $_{A^{*}}$ in $\mathcal{R} \mathcal{M}_{2}^{\mathrm{P}}$ is also the $\mathcal{L}$-class of id $A^{*}$ in $\mathcal{R} \mathcal{M}_{2}^{\text {fin }}$.

Proposition 2.12 $\mathcal{R} \mathcal{M}_{2}^{\mathrm{P}}$ has trivial group of units, i.e., the $\mathcal{D}$-class of the identity $\mathrm{id}_{A^{*}}$ is $\mathcal{H}$-trivial.

Proof. If $f \equiv_{\mathcal{H}}$ id $_{A^{*}}$ then by Lemma2.11 (for the $\mathcal{L}$-class of id $A^{*}$ ), $f(x)=v x$ for all $x$. Also by Lemma 2.11 (for the $\mathcal{R}$-class of $\mathrm{id}_{A^{*}}$ ), $f\left(x_{1}\right)=v x_{1}=\varepsilon$, for some $x_{1}$. This implies $v=\varepsilon$, hence $f=\operatorname{id}_{A^{*}}$.

As a consequence of Lemma 2.11, $\mathcal{R} \mathcal{M}_{2}^{\mathrm{P}}$ can be injectively mapped (non-homomorphically) into the $\mathcal{R}$-class of $\operatorname{id}_{A^{*}} \in \mathcal{R} \mathcal{M}_{2}^{\mathrm{P}}$. Let us define $f \mapsto \psi_{f}$ by $\operatorname{Dom}\left(\psi_{f}\right)=\{0\} \cup 1 \operatorname{Dom}(f)$, and

$$
\begin{aligned}
& \psi_{f}(0)=\varepsilon, \quad \text { and } \\
& \psi_{f}(1 x)=1 f(x), \text { for all } x \in \operatorname{Dom}(f) .
\end{aligned}
$$

Then for all $1 x \in 1 A^{*}, \quad \psi_{g \circ f}(1 x)=\left(\psi_{g} \circ \psi_{f}\right)(1 x)=1(f g)(x)$. So, $\left.f \mapsto \psi_{f}\right|_{1 A^{*}}$ is a morphism (where $\left.\psi_{f}\right|_{1 A^{*}}$ is the restriction of $\psi_{f}$ to $1 A^{*}$ ). But $\psi$ is not a morphism; indeed, since $\mathcal{R} \mathcal{M}_{2}^{\mathrm{P}}$ contains non-trivial groups, but the $\mathcal{D}$-class of $\mathrm{id}_{A^{*}}$ is $\mathcal{H}$-trivial, there cannot be a homomorphic embedding of $\mathcal{R} \mathcal{M}_{2}^{\mathrm{P}}$ into the $\mathcal{D}$-class of id $_{A^{*}}$.

\section{$3 \quad$ Embedding fP into $\mathcal{R} \mathcal{M}_{2}^{\mathrm{P}}$}

\section{Transforming any map into a right-ideal morphism:}

The semigroup fP uses the alphabet $\{0,1\}$; let \# be a new letter. For $f \in \mathrm{fP}$ we define $f_{\#}:\{0,1, \#\}^{*} \rightarrow$ $\{0,1, \#\}^{*}$ by letting $\operatorname{Dom}\left(f_{\#}\right)=\operatorname{Dom}(f) \#\{0,1, \#\}^{*}$, and

$$
f_{\#}(x \# w)=f(x) \# w
$$

for all $x \in \operatorname{Dom}(f)\left(\subseteq\{0,1\}^{*}\right)$, and all $w \in\{0,1, \#\}^{*}$. So $\operatorname{dom} C\left(f_{\#}\right)=\operatorname{Dom}(f) \#$.

\section{Proposition 3.1.}

(1) For any $L \subseteq\{0,1\}^{*}, L \#$ is a prefix code in $\{0,1, \#\}^{*}$.

(2) $L$ is in $\mathrm{P}$ iff $L \#$ is in $\mathrm{P}$.

(3) For any partial function $f:\{0,1\}^{*} \rightarrow\{0,1\}^{*}, f_{\#}$ is a right ideal morphism of $\{0,1, \#\}^{*}$.

(4) $f \in \mathrm{fP}$ iff $f_{\#} \in \mathcal{R} \mathcal{M}_{3}^{\mathrm{P}}$.

Proof. This is straightforward.

\section{Coding from three letters to two letters:}

We consider the following encoding from the 3-letter alphabet $\{0,1, \#\}$ to the 2 -letter alphabet $\{0,1\}$. 
code $:\{0,1, \#\} \rightarrow\{00,01,11\}$ is defined by

$\operatorname{code}(0)=00, \quad \operatorname{code}(1)=01, \quad \operatorname{code}(\#)=11$.

For a word $w \in\{0,1, \#\}^{*}, \operatorname{code}(w)$ is the concatenation of the encodings of the letters in $w$.

The choice of this code is somewhat arbitrary; e.g., we could have picked the encoding $c$ from $\{0,1, \#\}$ onto the maximal prefix code $\{00,01,1\}$, defined by $c(0)=00, c(1)=01, c(\#)=1$.

Definition 3.2 We define $f^{C}:\{0,1\}^{*} \rightarrow\{0,1\}^{*}$ by letting $\operatorname{Dom}\left(f^{C}\right)=\operatorname{code}(\operatorname{Dom}(f) \#)\{0,1\}^{*}$, and

$$
f^{C}(\operatorname{code}(x \#) v)=\operatorname{code}(f(x) \#) v
$$

for all $x \in \operatorname{Dom}(f)\left(\subseteq\{0,1\}^{*}\right)$, and all $v \in\{0,1\}^{*}$. We call $f^{C}$ the encoding of $f$.

\section{Proposition 3.3 .}

(1) For any $L \subseteq\{0,1\}^{*}$, $\operatorname{code}(L \#)$ is a prefix code.

(2) $L$ is in $\mathrm{P}$ iff $\operatorname{code}(L \#)$ is in $\mathrm{P}$.

(3) For any partial function $f:\{0,1\}^{*} \rightarrow\{0,1\}^{*}, f^{C}$ is a right ideal morphism of $\{0,1\}^{*}$.

(4) $f \in \mathrm{fP}$ iff $f^{C} \in \mathcal{R} \mathcal{M}_{2}^{\mathrm{P}}$.

Proof. This is straightforward.

\section{Proposition 3.4 .}

(1) The transformations $f \in \mathrm{fP} \longmapsto f_{\#} \in \mathcal{R} \mathcal{M}_{3}^{\mathrm{P}}$ and $f \in \mathrm{fP} \longmapsto f^{C} \in \mathcal{R} \mathcal{M}_{2}^{\mathrm{P}}$ are injective total homomorphisms from $\mathrm{fP}$ into $\mathcal{R} \mathcal{M}_{3}^{\mathrm{P}}$, respectively $\mathcal{R} \mathcal{M}_{2}^{\mathrm{P}}$.

(2) $f$ is regular in $\mathrm{fP}$ iff $f_{\#}$ is regular in $\mathcal{R} \mathcal{M}_{3}^{\mathrm{P}}$ iff $f^{C}$ is regular in $\mathcal{R} \mathcal{M}_{2}^{\mathrm{P}}$.

(3) There are one-to-one correspondences between the inverses of $f$ in $\mathrm{fP}$, the inverses of $f_{\#}$ in $\mathcal{R} \mathcal{M}_{3}^{\mathrm{P}}$, and the inverses of $f^{C}$ in $\mathcal{R} \mathcal{M}_{2}^{\mathrm{P}}$.

Proof. (1) is straightforward, and (2) follows from injectiveness and from the fact that the homomorphic image of an inverse is an inverse.

(3) Let $G \in \mathcal{R} \mathcal{M}_{3}^{\mathrm{P}}$ be such that $f_{\#} \circ G \circ f_{\#}=f_{\#}$; i.e., $f_{\#}(G(f(x) \# w))=f(x) \# w$, for all $x \in\{0,1\}^{*}$ and $w \in\{0,1, \#\}^{*}$. Since $f_{\#}(G(f(x) \# w))(=f(x) \# w)$ contains $\#, G(f(x) \# w)$ is of the form $G(f(x) \# w)=z \# v$, for some $z \in\{0,1\}^{*}$ and $v \in\{0,1, \#\}^{*}$. Hence $f_{\#}(G(f(x) \# w))=$ $f_{\#}(z \# v)=f(z) \# v=f(x) \# w$, so $f(z)=f(x)$ and $v=w$. So, $G(f(x) \# w)=z \# w$ for some $z \in f^{-1} f(x)$. Thus there exists a function $g:\{0,1\}^{*} \rightarrow\{0,1\}^{*}$ such that $G(y \#)=g(y) \#$ for all $y \in \operatorname{Im}(f)$; then $G(y \# w)=g(y) \# w$, for all $w \in\{0,1, \#\}^{*}$. Hence $g$ is an inverse of $f$. Moreover, $g$ is clearly in fP if $G$ is in $\mathcal{R} \mathcal{M}_{3}^{\mathrm{P}}$.

Let $H \in \mathcal{R} \mathcal{M}_{2}^{\mathrm{P}}$ be such that $f^{C} \circ H \circ f^{C}=f^{C}$; i.e., $f^{C}(H(\operatorname{code}(f(x) \#) v))=\operatorname{code}(f(x) \#) v$, for all $x, v \in\{0,1\}^{*}$. Since $f^{C}$ outputs $\operatorname{code}(f(x) \#) v$ on input $H(\operatorname{code}(f(x) \#) v)$, the definition of $f^{C}$ implies that for all $v \in\{0,1\}^{*}: H(\operatorname{code}(f(x) \#) v)$ is of the form $\operatorname{code}(z \#) v$ for some $z \in\{0,1\}^{*}$ with $f(z)=f(x)$. Hence there exists a function $h:\{0,1\}^{*} \rightarrow\{0,1\}^{*}$ such that $H(\operatorname{code}(y \#))=\operatorname{code}(h(y) \#)$ for all $y$; then $H(\operatorname{code}(y \#) v)=\operatorname{code}(h(y) \#) v$, for all $y, v$. Hence $h$ is an inverse of $f$. Moreover, $h$ is clearly in $\mathrm{fP}$ if $H$ is in $\mathcal{R} \mathcal{M}_{2}^{\mathrm{P}}$.

We will show in Section 5 that the encoding $f \mapsto f^{C}$ corresponds to an "inversive reduction".

In summary we have the following relation between $\mathrm{fP}$ and $\mathcal{R} \mathcal{M}_{2}^{\mathrm{P}}$ :

$$
\mathrm{fP} \stackrel{C}{\hookrightarrow} \mathcal{R} \mathcal{M}_{2}^{\mathrm{P}} \hookrightarrow[\mathrm{id}]_{\mathcal{J}(\mathrm{fP})}^{0} \hookrightarrow \mathrm{fP} .
$$

Here $[\mathrm{id}]_{\mathcal{J}(\mathrm{fP})}^{0}$ is the $\mathcal{J}$-class of the identity id of $\mathrm{fP}$, together with the zero element (i.e., it is the Rees quotient of the $\mathcal{J}$-class of id in fP). The embedding into [id $]_{\mathcal{J}(\mathrm{PP})}^{0}$ holds because $\mathcal{R} \mathcal{M}_{2}^{\mathrm{P}}$ is $\mathcal{J}^{0}$-simple. 


\section{Evaluation maps}

The Turing machine evaluation function eval ${ }_{\mathrm{TM}}$ is the input-output function of a universal Turing machine; it has the form $\mathrm{eval}_{\text {тм }}(w, x)=\phi_{w}(x)$, where $\phi_{w}$ is the input-output function described by the word ("program") $w$. (Recall that by "function" we always mean partial function.) Similarly, there is an evaluation function for acyclic circuits, eval circ $(C, x)=f_{C}(x)$, where $f_{C}$ is the input-output map of the circuit $C$. Here we will only consider length-preserving circuits, i.e., $\left|f_{C}(x)\right|=|x|$. We also identify the circuit with a bitstring that describes the circuit. The map eval circ $_{\text {is }}$ polynomial-time computable, but not polynomially balanced (since the size of input component $C$ is not bounded in terms of the output length $\left.\left|f_{C}(x)\right|\right)$.

Levin [18] noted that functions of the form

$$
\mathrm{ev}(w, x)=\left(w, \phi_{w}(x)\right)
$$

(under some additional assumptions) are polynomially balanced and polynomial-time computable; and he observed that ev is a critical one-way function in the following sense:

Definition 4.1 A function $e \in \mathrm{fP}$ is critical (or fP-critical) iff the following holds: One-way functions exist iff the function e is a one-way function. Similarly, a set $L \in N P$ is critical (or P-critical) iff the following holds: $\mathrm{P} \neq \mathrm{NP}$ iff $L \notin \mathrm{P}$.

The literature calls these functions "universal" one-way functions; however, not all critical one-way functions are universal (in the sense of universal Turing machines, or other universal computing devices). Levin's idea of a "universal" (critical) one-way function has also been used in probabilistic settings for one-way functions (see e.g., [12]).

To make ev polynomial-time computable, additional features have to be introduced. One approach is to simply build a counter into the program of ev that stops the computation of ev $(w, x)$ after a polynomial number of steps (for a fixed polynomial). For example, the computation could be stopped after a quadratic number of steps, i.e., $c(|w|+|x|)^{2}+c$ steps (for a fixed constant $c \geq 1$ ); we call this function $\mathrm{ev}_{(2)}$. There exist other approaches; see e.g., section 2.4 of [12], or p. 178 of [1], where it is proved that $\mathrm{ev}_{(2)}$ is $\mathrm{fP}$-critical.

Here is another simple example of a critical one-way function:

$$
\mathrm{ev}_{\text {circ }}(C, x)=\left(C, f_{C}(x)\right) \text {, }
$$

where $C$ ranges over finite acyclic circuits (more precisely, strings that describe finite acyclic circuits), and $f_{C}$ is the input-output map of a circuit $C$. The function ev circ $_{\text {in }}$ in fP; it is balanced since $|x| \leq|C|$ and $C$ is part of the output. Here we only consider length-preserving circuits, i.e., $\left|f_{C}(x)\right|=|x|$. We will prove later that $\mathrm{ev}_{\text {circ }}$ is not only critical, but also complete with respect to a reduction that is appropriate for one-way functions.

A similar example of a critical function is $(B, \tau) \longmapsto(B, B(\tau))$, where $B$ ranges over all boolean formulas (or over all boolean formulas in $3 \mathrm{CNF}$ ), $\tau$ is any truth-value assignment for $B$ (i.e., a bitstring whose length is the number of boolean variables in $B$ ), and $B(\tau)$ is the truth-value of $B$ for the truthvalue assignment $\tau$. More generally we have:

Proposition 4.2 For a nondeterministic polynomial-time Turing machine $M$, let $f_{M}$ be defined by $f_{M}(x, s)=x$ iff $M$, with choice sequence $s$, accepts $x$ (and undefined otherwise).

Then $f_{M}$ is $\mathrm{fP}$-critical iff the language (in NP) accepted by $M$ is $\mathrm{P}$-critical.

Proof. We studied the functions $f_{M}$ in Prop. 1.9 (where we used the notation $f_{L}$ ). We saw that $f_{M}$ is one-way iff $\operatorname{Im}\left(f_{M}\right) \notin \mathrm{P}$. Moreover, $\operatorname{Im}\left(f_{M}\right)$ is the language accepted by $M$, and $\operatorname{Im}\left(f_{M}\right) \in \mathrm{NP}$. So, $f_{M}$ is one-way iff $\mathrm{P} \neq \mathrm{NP}$. 


\section{Machine model for fP:}

Every function in fP can be computed by a Turing machine with a built-in polynomial-time counter, that is used for enforcing time-complexity and input balance. As usual, to say that time-complexity or balance functions are "polynomial" means that they have polynomial upper-bounds. More precisely, we will describe every polynomial-time multi-tape Turing machine $M$ by a program $v$ (which consists of the list of transitions of the Turing machine, as well as its start and accept states), and a polynomial $p$ such that $p(n)$ is an upper-bound on the time-complexity and the input balance of $M$ on all inputs of length $\leq n$. Since we only require polynomial upper-bounds, we can take $p$ of the form $p(n)=a n^{k}+a$, where $k, a$ are positive integers. So $p$ is determined by two integers (stored as bitstrings). We do not need to assume anything about the time-complexity of the Turing machine with program $v$ (and in general it is undecidable whether $v$ has polynomial-time complexity); instead, we want to consider pairs $(v, p)$ where $v$ is a Turing machine program, and $p$ is a polynomial (given by two integers $k, a$ ). Based on pairs $(v, p)$ we define the following: A partial function $f$ is computed by $(v, p)$ iff for all $x \in A^{*}, f(x)$ is computed by the Turing machine with program $v$ in time $\leq a|x|^{k}+a=p(|x|)$ and input balance $\leq p(|f(x)|)$; when $f(x)$ is undefined then the program either gives no output, or violates the time bound or the input balance bound. In this way fP can be recursively enumerated by pairs $(v, p)$. For $\mathrm{P}$ and NP this (or a similar idea) goes back to the work of Hartmanis, Lewis, Stearns, and others in the 1970's; compare with the generic NP-complete problem in [14, the proof of the complexity hierarchy theorems in chapter 12 in [16], and the section on critical ("universal") one-way functions in [12].

However, pairs $(v, p)$ do not form a machine model, being hybrids consisting of a machine and two numbers. In order to obtain a machine model for $\mathrm{fP}$ we take a Turing machine with program $v$, and add an extra tape that will be used as a counter. We assume that every tape has a left endmarker. On input $x$, a Turing machine with counter first computes $p(|x|)=a|x|^{k}+a$, and moves the head of the counter tape $p(|x|)$ positions to the right. After the counter has been prepared (and the head on the input $x$ has been moved back to the left end), the Turing machine executes program $v$ on the other tapes, while in each transition the head on the counter tape moves left by one position. If the counter head gets back to the left endmarker ("it triggers the counter"), the Turing machine stops and rejects (and produces no output). If the machine halts before triggering the counter, the counter has no effect on the result of program $v$ on input $x$. After this, if there is an output $y$ the Turing machine with counter checks the input balance: If $|y| \geq|x|$ the balance condition obviously holds, so $y$ is the final output. If $|y|<|x|$, the machine computes $p(|y|)(<p(|x|))$; if $|x|>p(|y|)$ the machine rejects (and produces no final output); otherwise, $y$ is the final output.

In order to mark off space of length $p(|x|)$ on the counter tape, we need an algorithm for computing $p(|x|)=a|x|^{k}+a$, and we will look at the time-complexity of this algorithm. Recall that the bitlength of a positive integer $n$ is $\left\lfloor\log _{2} n\right\rfloor+1$ (in unsigned binary representation).

(1) First, we compute $|x|$ in binary, by repeatedly dividing $|x|$ by 2 , using two tapes: On one tape, we start with a length $n=|x|$, then a mod-2 counter produces $\lfloor n / 2\rfloor$ on the 2 nd tape and records the remainder ( 0 or 1 ) on a 3rd tape; then a mod-2 counter computes half of the 2nd tape and writes it on the 1st tape, and records the remainder on tape 3 , etc. This takes time $\leq \sum_{i=0}^{\left\lfloor\log _{2}|x|\right\rfloor}|x| / 2^{i}$ $=2^{\left.\log _{2}|x|\right\rfloor+1}-1<2|x|$.

(2) We compute $a|x|^{k}+a$ in binary, using $k$ multiplications and one addition. This takes time

$$
\begin{aligned}
\leq & c\left(\log _{2}|x|\right)^{2}+2 c\left(\log _{2}|x|\right)^{2}+\ldots+(k-1) c\left(\log _{2}|x|\right)^{2}+c k \log _{2}|x| \log _{2} a \\
& +c \max \left\{k \log _{2}|x|, \log _{2} a\right\} \\
< & c k^{2}\left(\log _{2}|x|\right)^{2}+c k \log _{2} a+c k \log _{2}|x| \quad\left(\text { when } k \log _{2}|x| \geq \log _{2} a, \text { i.e., }|x| \geq a^{1 / k}\right) \\
< & c k^{2} \log _{2} a\left(\log _{2}|x|\right)^{2},
\end{aligned}
$$

where $c$ is a positive constant that depends on the details of the multiplication and addition algorithms. Two integers $n_{1}, n_{2}$ (in binary) can be multiplied in time $\leq c \log _{2} n_{1} \log _{2} n_{2}$, and added in time $\leq c \max \left\{\log _{2} n_{1}, \log _{2} n_{2}\right\}$. The bitlength of the product $n_{1} n_{2}$ is $\leq\left\lfloor\log _{2} n_{1}\right\rfloor+\left\lfloor\log _{2} n_{2}\right\rfloor+2$. For the 
last expression in the calculation above, we have $c k^{2} \cdot \log _{2} a \cdot\left(\log _{2}|x|\right)^{2}<|x|$ for large enough $|x|$, i.e., when $c_{p} \leq|x|$ (where $c_{p}$ is a positive integer depending on $p$ ).

Remark (concrete upper-bound on $c_{p}$ ): We define $c_{p}$ to be the smallest number $N$ such that for all $x \in A^{\geq N}$, the time to prepare the counter is $\leq|x|$. We saw that this time is $\leq|x|$ when $|x| \geq a^{1 / k}$ and $|x| \geq c k^{2} \log _{2} a\left(\log _{2}|x|\right)^{2}$. One proves easily that $n \geq\left(\log _{2} n\right)^{2}$ for all $n \geq 16$. So, $|x|=|x|^{1 / 2} \cdot|x|^{1 / 2}$ $\geq c k^{2} \log _{2} a \cdot\left(\log _{2}|x|\right)^{2}$ is implied by $|x|^{1 / 2} \geq c k^{2} \log _{2} a$ and $|x|^{1 / 2} \geq 16$. Thus we have: $c_{p} \leq$ $\max \left\{256, c^{2} k^{4}\left(\log _{2} a\right)^{2}, a^{1 / k}\right\}$.

(3) We mark off space of length $p(|x|)$ by converting the binary representation of $p(|x|)$ to "unary": This is done by the Horner scheme with repeated doubling (where the doubling is done by using two tapes, and writing two spaces on the 2 nd tape for each space on the 1st tape). This takes time $\sum_{i=0}^{\left\lfloor\log _{2} p(|x|)\right\rfloor+1} 2^{i}<4 p(|x|)$.

Thus, the total time used to prepare the counter on input $x$ is $<2|x|+|x|+4 p(|x|) \leq 7 p(|x|)$, when $|x| \geq c_{p}$.

For inputs $x$ with $|x|<c_{p}$, the counter will also receive space $p(|x|)$, but the time used for this could be more than $7 p(|x|)$. We can remove the exception of the finitely many inputs of length $<c_{p}$ as follows. For these inputs we let the Turing machine operate as a finite-state machine (without using the work tapes or any counter); for such an input $x$, the time to set up the counter will then be $\leq|x|+p(|x|)(<7 p(|x|))$.

After execution of program $v$ on input $x$ for time $\leq p(|x|)$, if there is an output $y$ so far, the input balance is checked. If $|y| \geq|x|$ input balance holds automatically; checking whether $|y| \geq|x|$ takes time $\leq|x|$. If $|x|>|y|$, we compute $p(|y|)(<p(|x|))$ in binary, in the same ways as in steps (1) and (2) of the counter-tape preparation above. This takes time $\leq 2|y|+|y|<3|x|$, when $|y| \geq c_{p}$. The time needed to compare the binary representations of $|x|$ and $|y|$ (of length $\leq\left\lfloor\log _{2}|x|\right\rfloor+1$ ) is absorbed in the time to calculate $p(|y|)$. So, the time for checking the input balance is $<|x|+3|x|<4 p(|x|)$.

So far we have obtained a machine, described by $(v, p)$, whose time-complexity is $\leq 7 p()+$. $p()+.4 p()=.12 p($.$) and whose input balance is \leq p($.$) . Because of the preparation of the counter,$ the time-complexity of the new machine is always larger than $p($.$) ; therefore we will further modify$ the construction, as follows. Let $p(n)=a\left(n^{k}+1\right)$; we will assume from now on that $a \geq 12$. Let $p^{\prime}(n)=(a-a \% 12) \cdot\left(n^{k}+1\right)$, where $a \% 12$ is the remainder of the division of $a$ by 12 . So, $a-a \% 12 \geq 12$ and $a-a \% 12$ is a multiple of 12 . (1) Instead of marking off space of length $p(|x|)$, the new machine marks off length $\frac{1}{12} p^{\prime}(|x|)$, in time $\leq \frac{7}{12} p^{\prime}(|x|)$. (2) It executes the program $v$ for time $\leq \frac{1}{12} p^{\prime}(|x|)$, using the marked-off counter. (3) It checks the input balance in time $\leq \frac{4}{12} p^{\prime}(|x|)$. The total time of the modified machine is then $\leq p^{\prime}(|x|) \leq p(|x|)$, and the input balance is $\leq p^{\prime}(|x|) \leq p(|x|)$.

So for every Turing machine program $v$ and any polynomial $p$ this modified machine computes a function in $\mathrm{fP}$. Conversely, if $v$ is a program with polynomial time for a function $f \in \mathrm{fP}$, then the modified program with pair $(v, p)$ correctly computes $f$ if $\frac{1}{12} p^{\prime}($.$) is larger than the time (and balance)$ that $v$ uses on all inputs; if $f$ belongs to fP then a polynomial $p$ such that $\frac{1}{12} p^{\prime}($.$) is large enough$ for bounding the time and the input balance, exists. Such a modified machine will be called Turing machine with polynomial counter. A program for such a machine consists of a pair $(v, p)$ and an extra program for preparing the counter and checking input balance; let's call that extra program $u_{p}$ (it only depends on $p$, and not on $v)$. The triple $\left(v, p, u_{p}\right)$, or more precisely, the word code $\left(v \# k \# a \# u_{p}\right)$ with the numbers $k, a$ written in binary, will be called a polynomial program. Thus, Turing machines with polynomial counter are a machine model for fP. So we have proved:

Proposition 4.3 There exists a class of modified Turing machines, called Turing machine with polynomial counter, with the following properties: For every $f \in \mathrm{fP}$ there exists a Turing machine with polynomial counter that computes $f$; and for every Turing machine with polynomial counter, the inputoutput function belongs to fP.

Notation: A polynomial program $w=\operatorname{code}\left(v \# k \# a \# u_{p}\right)$, based on a Turing machine program $v$ and a polynomial $p$ (with $\left.p(n)=a n^{k}+a\right)$, will be denoted by $\langle v, p\rangle$. The polynomial $p$ that appears 
in $w$ will often be denoted by $p_{w}$. The function computed by a polynomial program $w=\langle v, p\rangle$ will be denoted by $\phi_{w}(\in \mathrm{fP})$.

\section{Evaluation maps for fP:}

At first we consider a function ev poly defined by $\mathrm{ev}_{\text {poly }}(w, x)=\left(w, \phi_{w}(x)\right)$, where $w=\langle v, p\rangle$ is any polynomial program. But ev poly is not in fP. Indeed, the output length (and hence the time-complexity) of ev poly on input $(w, x)$ is equal to $p(|x|)$ (in infinitely many cases, when $p$ is a tight upper-bound); as $w$ varies, the degree of $p$ is unboundedly large, hence the time-complexity of ev poly has no polynomial upper-bound. We will nevertheless be able to build fP-critical functions. For a fixed polynomial $q$ (of the form $\left.q(n)=a n^{k}+a\right)$, let

$$
\mathrm{fP}^{q}=\left\{\phi_{w} \in \mathrm{fP}: p_{w} \leq q\right\} .
$$

More explicitly, $p_{w} \leq q$ means that the polynomial program $w$ has time-complexity $\leq p_{w}(|x|) \leq q(|x|)$ and input-balance $|x| \leq p_{w}\left(\left|\phi_{w}(x)\right|\right) \leq q\left(\left|\phi_{w}(x)\right|\right)$, for all $x \in \operatorname{Dom}\left(\phi_{w}\right)$.

In general, for polynomials $q_{1}, q_{2}$ we say $q_{1} \leq q_{2}$ iff for all non-negative integers $n$ : $q_{1}(n) \leq q_{2}(n)$. Interestingly, for polynomials of the form $q_{i}(n)=a_{i} n^{k_{i}}+a_{i}$ we have: $q_{1} \leq q_{2}$ iff $k_{1} \leq k_{2}$ and $a_{1} \leq a_{2}$. Hence it is easy to check whether $p_{w} \leq q$, given $w$ and the two numbers that determine $q$.

A polynomial program $w$ such that $p_{w} \leq q$ (for a fixed polynomial $q$ ) is called a $q$-polynomial program. We define $\mathrm{ev}_{q}$ by

$$
\mathrm{ev}_{q}(w, x)=\left(w, \phi_{w}(x)\right)
$$

where $w$ is any $q$-polynomial program. The function $\mathrm{ev}_{q}$ above has two input and two output strings. To make $\mathrm{ev}_{q}$ fit into our framework of functions with one input and one output string we encode $\mathrm{ev}_{q}$ as $\operatorname{ev}_{q}^{C}:\{0,1\}^{*} \rightarrow\{0,1\}^{*}$ where for all $w, x \in\{0,1\}^{*}$ such that $w$ is a $q$-polynomial program,

$$
\operatorname{ev}_{q}^{C}(\operatorname{code}(w) 11 x)=\operatorname{code}(w) 11 \phi_{w}(x) .
$$

From now on we will call $\mathrm{ev}_{q}^{C}$ an "evaluation map". We observe that in the special case where $\phi_{w}$ (for a fixed $w$ ) is a right ideal morphism, the function

$$
\operatorname{ev}_{q}^{C}(\operatorname{code}(w) 11 \cdot): \quad x \longmapsto \operatorname{code}(w) 11 \phi_{w}(x)
$$

is also a right ideal morphism.

\section{Criticality of $\mathrm{ev}_{q}^{C}$ :}

For any fixed word $v \in\{0,1\}^{*}$ we define the prepending map

$$
\pi_{v}: x \in\{0,1\}^{*} \longmapsto v x
$$

and for any fixed positive integer $k$ we define

$$
\pi_{k}^{\prime}: z x \in\{0,1\}^{*} \longmapsto x \text {, where }|z|=k \text { (with } \pi_{k}^{\prime}(t) \text { undefined when }|t|<k \text { ). }
$$

Clearly, $\pi_{v}, \pi_{k}^{\prime} \in \mathcal{R} \mathcal{M}_{2}^{\mathrm{P}}$, and we have $\pi_{|v|}^{\prime} \circ \pi_{v}=\mathrm{id}_{A^{*}}$ (i.e., $\pi_{|v|}^{\prime}$ is a left inverse of $\pi_{v}$ ).

We observe that $\pi_{v}$ can be written as a composite of the maps $\pi_{0}$ and $\pi_{1}$, for any $v \in\{0,1\}^{*}$. Similarly, $\pi_{k}^{\prime}$ is the $k$ th power of $\pi_{1}^{\prime}$.

Proposition 4.4 Let $q$ be any polynomial such that for all $n \geq 0, q(n)>c n+c$ (where $c>1$ is a constant). Then $\mathrm{ev}_{q}^{C}$ belongs to $\mathrm{fP}$, and $\mathrm{ev}_{q}^{C}$ is a one-way function if one-way functions exist.

Proof. We saw that testing whether $p_{w} \leq q$ is easy for polynomials of the form that we consider. By reviewing the workings of a universal Turing machine (e.g., in [16]) we see that the time-complexity of $\mathrm{ev}_{q}(w, x)$ is $\leq c_{0}|w| \cdot p_{w}(|x|)^{2}$ (when $p_{w}$ is at least linear); here, $c_{0} \geq 1$ is a constant (independent of $x$ and $w)$. The factor $p_{w}(|x|)^{2}$ comes the fact that Turing machines can have any number of tapes, whereas a Turing machine for $\mathrm{ev}_{q}$ has a fixed number of tapes; any number of tapes can be converted to one tape, but the complexity increases by a square (the more efficient Hennie-Stearns 
construction converts any number of tapes to two tapes, with a complexity increase from $T$ to $T \log T$ ). The universal Turing machine simulates each transition of program $w$ (modified into a 1-tape Turing machine) using $\leq c_{1}|w|$ steps (for a constant $c_{1} \geq 1$ ).

For input balance: When $|x| \leq\left|\phi_{w}(x)\right|$ we also have $|w|+|x| \leq|w|+\left|\phi_{w}(x)\right|$ so balance is automatic. When $|x|>\left|\phi_{w}(x)\right|$ then (since $p_{w}$ bounds the input balance of $\phi_{w}$ ), the input-length satisfies $|w|+|x| \leq|w|+p_{w}\left(\left|\phi_{w}(x)\right|\right) \leq|w|+q\left(\left|\phi_{w}(x)\right|\right) \leq q\left(|w|+\left|\phi_{w}(x)\right|\right)$. Hence $\mathrm{ev}_{q}$, and similarly $\mathrm{ev}_{q}^{C}$, belongs to fP.

Criticality: If the function $\mathrm{ev}_{q}$ has an inverse $e_{q}^{\prime} \in \mathrm{fP}$, then $\operatorname{ev}_{q} \circ e_{q}^{\prime} \circ \mathrm{ev}_{q}(w, x)=\left(w, \phi_{w}(x)\right.$. Hence, for any function $\phi_{w} \in \mathrm{fP}^{q}$ with a fixed program $w$ we have: $\phi_{w} \circ \pi_{|w|}^{\prime} \circ e_{q}^{\prime} \circ \pi_{w} \circ \phi_{w}=\phi_{w}$, where $\pi_{|w|}^{\prime}:(w, v) \mapsto v$, and $\pi_{w}: y \mapsto(w, y)$. For a fixed $w$ we have $\pi_{|w|}^{\prime}, \pi_{w} \in \mathrm{fP}$, so $\pi_{|w|}^{\prime} \circ e_{q}^{\prime} \circ \pi_{w}$ is an inverse of $\phi_{w}$. Hence, if $\mathrm{ev}_{q}$ is not one-way, no function in $\mathrm{fP}^{q}$ is one-way. But there are functions (e.g., some $f_{M}$ as seen in Prop. 4.2) that are fP-critical, even when $q$ is linear (since by padding arguments one can obtain NP-complete languages with nondeterministic linear time-complexity). So some fP-critical $f_{M}$ would have an inverse in $\mathrm{fP}$. The same proof is easily adapted to $\mathrm{ev}_{q}^{C}$.

\section{Finite generation of $\mathrm{fP}$ :}

We will show that $\mathrm{ev}_{q}^{C}$ can be used to simulate universal evaluation maps, and to prove that fP is finitely generated. This is based on the universality of $\mathrm{ev}_{q_{2}}^{C}$ for $\mathrm{fP}^{q_{2}}$, combined with a padding argument; here $q_{2}$ is a polynomial of degree 2 , with $q_{2}(n) \geq c n^{2}+c$ for a constant $c \geq 12$. (We chose 12 in view of the reasoning before Prop. 4.3.) We need some auxiliary functions first.

We define the expansion (or padding) map, first as a multi-variable function for simplicity:

$$
\operatorname{expand}(w, x)=\left(\mathrm{e}(w),\left(0^{4}|x|^{2}+7|x|+2, x\right)\right)
$$

where $\mathrm{e}(w)$ is such that

$$
\phi_{\mathrm{e}(w)}\left(0^{k}, x\right)=\left(0^{k}, \phi_{w}(x)\right), \text { for all } k \geq 0 .
$$

The program e $(w)$ is easily obtained from the program $w$, since it just processes the padding in front of the input and in front of the output of $\phi_{w}$, and acts as $\phi_{w}$ on $x$; and the time and balance polynomial is decreased due to padding. As a one-variable function, expand is defined by

$$
\text { expand }(\operatorname{code}(w) 11 x)=\operatorname{code}(\operatorname{ex}(w)) 110^{4|x|^{2}+7|x|+2} 11 x
$$

where for one-variable functions, the program ex $(w)$ is such that

$$
\phi_{\mathrm{ex}(w)}\left(0^{k} 11 x\right)=0^{k} 11 \phi_{w}(x) .
$$

Again, ex $(w)$ is a slight modification of $w=\operatorname{code}\left(v \# k \# a \# u_{p}\right)$ (following the notation for the machine model for fP), to allow inputs and outputs with padding, and to readjust the complexity and balance polynomial. More precisely, ex $(w)$ is of the form code $\left(r \# v \#\lceil k / 2\rceil \# a_{e} \# u_{p_{e}}\right)$, where $r$ is a preprocessing subprogram by which the prefix $0^{k} 11$ of the input is simply copied to the output; at the end of execution of $r$, the state and head-positions are the start state and start positions of the subprogram $w$. The appropriate complexity and balance polynomial stored in ex $(w)$ is

$$
p_{e}(n)=a_{e} n^{\lceil k / 2\rceil}+a_{e}, \text { with } a_{e}=\max \left\{12,\left\lceil a / 2^{k}\right\rceil+1\right\} .
$$

Indeed, if $m=|x|$, an input $0^{4|x|^{2}+7|x|+2} 11 x$ of $\phi_{\operatorname{ex}(w)}$ has length $i=4 m^{2}+8 m+4$. So, $m=\sqrt{i} / 2-1$. Let $a\left(m^{k}+1\right)$ be the polynomial of program $w$. The complexity of $\phi_{\mathrm{ex}(w)}$ on its input is $4 m^{2}+7 m+4$ (for reading the part $0^{*} 11$ of the input), plus $a\left(m^{k}+1\right)$ (for using $x$ and computing $\phi_{w}(x)$ ). So in terms of its input length $i$, the complexity of $\phi_{\mathrm{ex}(w)}$ is $<i+a\left(m^{k}+1\right) \leq i^{\lceil k / 2\rceil}+a\left((\sqrt{i} / 2-1)^{k}+1\right)$ $\leq i^{\lceil k / 2\rceil}+a / 2^{k} i^{k / 2}$; the last step uses the fact that $(z-1)^{m} \leq z^{m}-1$ for all $z \geq 0, m \geq 1$. Hence the complexity of $\phi_{\mathrm{ex}(w)}$ is $<\left(a / 2^{k}+1\right) i^{\lceil k / 2\rceil}$. For the input balance of $\phi_{\mathrm{ex}(w)}$ we have: The input-length is bounded by twice the output-length. Indeed, the input length is $i=4|x|^{2}+8|x|+4$ $<2 \cdot\left|0^{4|x|^{2}+7|x|+2} 11\right|<2 \cdot\left|0^{4|x|^{2}+7|x|+2} 11 \phi_{w}(x)\right|=2 \cdot\left|\phi_{\operatorname{ex}(w)}\left(0^{4|x|^{2}+7|x|+2} 11 x\right)\right|$. Moreover, we want $a_{e}$ to stay $\geq 12$ (in view of the reasoning before Prop. 4.3). 
In order to achieve an arbitrarily large polynomial amount of padding we iterate the quadratic padding operation. Therefore we define a repeated expansion (or re-padding) map, first as a twovariable function:

$$
\text { reexpand }\left(u,\left(0^{h}, x\right)\right)=\left(\mathrm{e}(u),\left(0^{4 h^{2}+8 h+2}, x\right)\right), \text { for all } h>0,
$$

where $\mathrm{e}($.$) is as above. As a one-variable function,$

$$
\text { reexpand }\left(\operatorname{code}(u) 110^{h} 11 x\right)=\operatorname{code}(\operatorname{ex}(u)) 110^{4 h^{2}+8 h+2} 11 x, \text { for any } h \geq 0,
$$

with ex(.) as in expand above.

We also introduce a contraction (or unpadding) map, which is a partial left inverse of expand. We define contr first as a multi-variable function:

$$
\operatorname{contr}\left(w,\left(0^{h}, y\right)\right)=(\mathrm{c}(w), y), \quad \text { if } h \leq 4|y|^{2}+7|y|+2 \quad \text { (undefined otherwise). }
$$

As a one-variable function, contr is defined by

$$
\operatorname{contr}\left(\operatorname{code}(w) 110^{h} 11 y\right)=\operatorname{code}(\operatorname{co}(w)) 11 y, \quad \text { if } h \leq 4|y|^{2}+7|y|+2
$$

(undefined otherwise).

The program transformations $\mathrm{c}($.$) and \mathrm{co}($.$) are inverses of e(.), respectively ex(.). So, c(.) and \mathrm{co}($. erase the prefix $r$ in ex $(u)$, and replace the polynomial $b n^{h}+b$, encoded in ex $(u)$, by $b_{c} n^{2 h}+b_{c}$, where $b_{c}=(b-1) 2^{2 h}$.

To invert repeated padding we introduce a repeated contraction (or unpadding) map, first as a multi-variable function. Note that if $h=4 k^{2}+8 k+2$ (which is the amount of padding introduced by reexpand), then $k=\frac{1}{2} \sqrt{h+2}-1$. Therefore, for any $h \geq 0$ we define

$$
\operatorname{recontr}\left(u,\left(0^{h}, y\right)\right)=\left(\mathrm{c}(u),\left(0^{\max \{1,\lfloor\sqrt{h+2} / 2\rfloor-1\}}, y\right)\right) \quad \text { (undefined on other inputs). }
$$

As a one-variable function, recontr is defined by

$$
\operatorname{recontr}\left(\operatorname{code}(u) 110^{h} 11 y\right)=\operatorname{code}(\operatorname{co}(u)) 110^{\max \{1,\lfloor\sqrt{h+2} / 2\rfloor-1\}} 11 y
$$

(undefined on other inputs).

The maps expand, reexpand, contr, and recontr belong to fP, and they are regular (they have polynomial-time inverses).

\section{Proposition 4.5 fP is finitely generated.}

Proof. We will show that the following is a generating set of fP:

$$
\text { \{expand, reexpand, contr, recontr, } \left.\pi_{0}, \pi_{1}, \pi_{1}^{\prime}, \mathrm{ev}_{q_{2}}^{C}\right\} \text {, }
$$

where $q_{2}$ is the polynomial $q_{2}(n)=c n^{2}+c$, with $c \geq 12$ (the number 12 comes from the discussion before Prop. 4.3).

Remark: The functions expand, reexpand, contr, and recontr all have quadratic time-complexity and balance functions, so they can be generated by $\pi_{0}, \pi_{1}, \pi_{1}^{\prime}$, and $\operatorname{ev}_{q_{2}}^{C}$ (provided that the constant $c$ in $q_{2}$ is chosen large enough, so that $\mathrm{ev}_{q_{2}}^{C}$ can execute expand, reexpand, contr, and recontr). Thus

$$
\left\{\pi_{0}, \pi_{1}, \pi_{1}^{\prime}, \mathrm{ev}_{q_{2}}^{C}\right\}
$$

is a generating set of $\mathrm{fP}$. We use the larger generating set since it yields simpler formulas.

Let $w$ be a program with polynomial counter and let $m$ be an integer upper-bound on $\log _{2}(a+k)$, where $p_{w}(n)=a n^{k}+a$. We also assume that the program $w=\langle v, p\rangle$ is such that for any polynomial $P()>.p_{w}(),.\langle v, P\rangle$ also computes $\phi_{w}$; indeed, since $\phi_{w} \in \mathrm{fP}$, we can choose $v$ and $p$ so that the execution of $v$ by the Turing machine with polynomial counter (described by $\langle v, p\rangle$ ) never triggers the counter; in that case, making the counter larger does not change the function. Then for all $x \in\{0,1\}^{*}$, 


$$
\phi_{w}(x)=\pi_{2\left|w^{\prime}\right|+2}^{\prime} \circ \text { contr } \circ \operatorname{recontr}^{2 m} \circ \mathrm{ev}_{q_{2}}^{C} \circ \operatorname{reexpand}^{2 m} \circ \text { expand } \circ \pi_{\text {code }(w) 11}(x),
$$

where $w^{\prime}=\operatorname{co}^{2 m+1} \circ \operatorname{ex}^{2 m+1}(w)$. Indeed,

$$
\begin{aligned}
& x \stackrel{\pi_{\text {code }(w)} 11}{\longmapsto} \operatorname{code}(w) 11 x \stackrel{\text { expand }}{\longmapsto} \operatorname{code}(\operatorname{ex}(w)) 110^{4|x|^{2}+7|x|+2} 11 x \\
& \stackrel{\text { reexpand }^{2 m}}{\longmapsto} \operatorname{code}\left(\mathrm{ex}^{2 m+1}(w)\right) 110^{N_{2 m+1}} 11 x .
\end{aligned}
$$

Here $N_{1}=|x|^{2}+7|x|+2$, and $\left|0^{N_{1}} 11 x\right|=(2(|x|+1))^{2}$; inductively, $N_{i}=4 N_{i-1}^{2}+8 N_{i-1}+2$ for $1<i \leq 2 m+1$, and $\left|0^{N_{i}} 11\right|=\left(2\left(N_{i-1}+1\right)\right)^{2}$.

Continuing the calculation,

$$
\begin{aligned}
& \stackrel{\operatorname{ev}_{q_{2}}^{C}}{\longmapsto} \operatorname{code}\left(\mathrm{ex}^{2 m+1}(w)\right) 110^{N_{2 m+1}} 11 \phi_{w}(x) \\
& \stackrel{\text { recontr }^{2 m}}{\longmapsto} \operatorname{code}\left(w^{\prime}\right) 110^{\ell} 11 \phi_{w}(x) \\
& \stackrel{\text { contr }_{\longmapsto}}{\longmapsto} \operatorname{code}\left(w^{\prime}\right) 11 \phi_{w}(x) \stackrel{\pi_{2\left|w^{\prime}\right|+2}^{\prime}}{\longmapsto} \phi_{w}(x)
\end{aligned}
$$

where $w^{\prime}=\mathrm{co}^{2 m+1} \circ \mathrm{ex}^{2 m+1}(w)$. We use $2 m$ in recontr ${ }^{2 m}$ because $\phi_{w}(x)$ could be much shorter than $x$ (but by input balance, $\left.|x| \leq p_{w}\left(\left|\phi_{w}(x)\right|\right)\right)$. As a consequence, we also use $2 m$ in reexpand ${ }^{2 m}$ in order to have equal numbers of program transformations ex(.) and co(.). Note that doing more input padding than necessary does not do any harm; also, if $w$ contains a polynomial $p_{w}$ larger than needed for computing $\phi_{w}$, this does not cause a problem (by our assumption on $v$ ). By the choice of $2 m$, the value of $\ell$ above is less than $4\left|\phi_{w}(x)\right|^{2}+7\left|\phi_{w}(x)\right|+2$, so contr can be applied correctly.

The argument of $\mathrm{ev}_{q_{2}}^{C}$ in the above calculation has length $>N_{2 m+1}+2+|x|$, which is much larger than the time it takes to simulate the machine with program $w$ on input $x$ (that time is $\left.<c_{0}|w| p_{w}(|x|)^{2}\right)$. In fact, by the choice of $m$, the polynomial encoded in $\operatorname{ex}^{2 m+1}(w)$ is the linear polynomial $12(n+1)$ (which is $<q_{2}(n)$ ). Hence, $\mathrm{ev}_{q_{2}}^{C}$ works correctly on its input in this context.

We saw that fP does not have an evaluation map in the same sense as the Turing evaluation map. However, formula $(\star)$ in the proof of Prop. 4.5 shows that the map $\operatorname{ev}_{q_{2}}^{C}$ simulates every function in $\mathrm{fP}$, in the following sense: $f_{2}$ simulates $f_{1}$ (denoted by $f_{1} \preccurlyeq f_{2}$ ) iff there exist $\beta, \alpha \in \mathrm{fP}$ such that $f_{1}=\beta \circ f_{2} \circ \alpha$; this is discussed further at the beginning of Section 5. Formula $(\star)$ in Prop. 4.5 implies:

Proposition 4.6 Every function $f \in \mathrm{fP}$ is simulated by $\mathrm{ev}_{q_{2}}^{C}$.

It follows from this and the definition of simulation that $\mathrm{ev}_{q_{2}}^{C}$ belongs to the $\mathcal{J}$-class of id $A^{*}$ in fP.

Since fP is finitely generated we now have two ways of representing each element $g \in \mathrm{fP}$ by a word: (1) We have $g=\phi_{w}$ for some polynomial program $w \in A^{*}$ (as seen in Prop. 4.4), and (2) $g$ can be represented by a string of generators (considering the finite set of generators of $\mathrm{fP}$ as an alphabet). The next proposition describes the translation between these two representations.

Proposition 4.7 There are total computable maps $\alpha, \beta$ such that for any word $s$ over a finite generating set of $\mathrm{fP}, \alpha(s)$ is a polynomial program for the function given by $s$; and for any polynomial program $u, \beta(u)$ is a word for $\phi_{u}$ over the generators of $\mathrm{fP}$.

More precisely, let $\Gamma$ be a finite generating set of $\mathrm{fP}$. For any $s \in \Gamma^{*}$, let $\Pi s \in \mathrm{fP}$ be the element of $\mathrm{fP}$ obtained by composing the generators in the sequence $s$. There exist total recursive "compiler maps" $\alpha: \Gamma^{*} \rightarrow\{0,1\}^{*}$ and $\beta:\{0,1\}^{*} \rightarrow \Gamma^{*}$ such that for all $s \in \Gamma^{*}$ and all $w \in\{0,1\}^{*}: f_{\alpha(s)}=\Pi s$, and $\Pi \beta(w)=\phi_{w}$.

Proof. The map $\beta$ is given by formula $(\star)$ in the proof of Prop. 4.5, where a representation over the generators is explicitly constructed. When $u$ is not a well-formed polynomial program we let $\beta(u)$ be a sequence of generators for the empty function. 
Conversely, by composing a sequence of generators, a function in fP is obtained (note that every sequence $s$ of generators has a finite length). More precisely, if $f_{1}, f_{2} \in \mathrm{fP}$ have as complexity and balance bounds the polynomials $q_{i}(n)=a_{i} n^{k_{i}}+a_{i}(i=1,2)$, then $f_{2} \circ f_{1}$ has input balance $\leq q_{2} \circ q_{1}(n)$ (obviously), and time-complexity $\leq q_{1}(n)+q_{2} \circ q_{1}(n)$. Indeed, a polynomial-time program for $f_{2} \circ f_{1}$ is obtained by first taking the program for $f_{1}$ on input $x$, and then applying the program for $f_{2}$ to $f_{1}(x)$ (in time $\leq q_{2}\left(\left|f_{1}(x)\right|\right)$ ). The corresponding polynomial upper-bound is $q_{1}(n)+q_{2} \circ q_{1}(n)=$ $a_{1}\left(n^{k_{1}}+1\right)+a_{2} a_{1}^{k_{2}}\left(n^{k_{1}}+1\right)^{k_{2}}+a_{2}<\left(a_{1}+a_{2} a_{1}^{k_{2}}\right)\left(n^{k_{1}}+1\right)^{k_{2}}+a_{2}$. In order to obtain a polynomial upper-bound of the form $a n^{k}+a$, we use the inequality

$$
(n+1)^{j} \leq 2^{j-1}\left(n^{j}+1\right), \text { for all } n \geq 0, j \geq 1 .
$$

(To prove this inequality apply calculus to the function $f(x)=2^{j-1}\left(x^{j}+1\right)-(x+1)^{j}$.) Thus for $f_{2} \circ f_{1}$ we get a complexity and balance upper-bound

$$
q(n)=a n^{k_{1} k_{2}}+a, \text { where } a=a_{2}+a_{1}+a_{2} a_{1}^{k_{2}} 2^{k_{2}} .
$$

This yields an algorithm for obtaining a polynomial program for $f_{2} \circ f_{1}$ from polynomial programs for $f_{1}$ and $f_{2}$. For a sequence of generators $s$, this algorithm can be repeated $|s|-1$ times to yield a polynomial programs for the sequence $s$ of generators.

A finite generating set $\Gamma$ for $\mathrm{fP}$ can be used to construct a generator-based evaluation map for $\mathrm{fP}$, defined by $(s, x) \in \Gamma^{*} \times A^{*} \longmapsto \mathrm{ev}_{\Gamma}(s, x)=(s,(\Pi s)(x))$. However, $\mathrm{ev}_{\Gamma}$ does not belong to $\mathrm{fP}$, for the same reasons as we saw at the beginning of Sect. 4 for ev poly. (But just as for ev poly we could restrict $\mathrm{ev}_{\Gamma}$ to a function that belongs to fP and that simulates every element of fP.)

Proposition 4.8 fP is not finitely presented. Its word problem is co-r.e., but not r.e.

Proof. The word problem is co-r.e.: Let $U, V \in \Gamma^{*}$; using Prop. 4.7 we effectively find programs $u, v \in A^{*}$ from $U, V$ such that $\phi_{u}=\Pi U, \phi_{v}=\Pi V$. If $\phi_{u} \neq \phi_{v}$ then by exhaustive search we will find $x$ such that $\phi_{u}(x) \neq \phi_{v}(x)$, thus showing that $U \neq V$ in $\mathrm{fP}$. When $U=V$ in $\mathrm{fP}$ then this procedure rejects by not halting.

The word problem of $\mathrm{fP}$ is undecidable, since the equality problem for languages in $\mathrm{P}$ can be reduced to this (reducing $L$ to $\mathrm{id}_{L}$ or to $\mathrm{id}_{\operatorname{code}(L \#)}$ ). And the equality problem for languages in $\mathrm{P}$ is undecidable, since the universality problem of context-free languages can be reduced to the equality problem for languages in $\mathrm{P}$; all context-free languages are in $\mathrm{P}$. The universality problem for contextfree language is the question whether for a given context-free grammar $G$ with terminal alphabet $A$ (with $|A| \geq 2$ ), the language generated by $G$ is $A^{*}$; this problem is undecidable (see [16] Thm. 8.11).

Since the word problem is co-r.e. but undecidable, it is not r.e. Hence these finitely generated monoids are not finitely presented (since the word problem of a finitely presented monoid is r.e.).

Proposition 4.9 fP is finitely generated by regular elements.

Proof. All the listed generators of fP are regular, except possibly $\mathrm{ev}_{q_{2}}^{C}$. Let us define a partial function $E_{q_{2}} \in \mathrm{fP}^{q_{2}}$ by $E_{q_{2}}(w, x)=\left(w, \phi_{w}(x), x\right)$, when $\phi_{w} \in \mathrm{fP}^{q_{2}}$. Obviously, $E_{q_{2}}$ is not one-way. But ev $q_{q_{2}}$ (as a two-variable function) can be expressed as a composition of $E_{q_{2}}$ and the other (regular) generators. In more detail, $\mathrm{ev}_{q_{2}}=\pi_{q_{2}}^{\prime} \circ E_{q_{2}}$, where $\pi_{q_{2}}^{\prime}(w, z, x)=(w, z)$ if $|z| \leq q_{2}(|x|)$ and $|x| \leq q_{2}(|z|)$. So $\mathrm{ev}_{q_{2}}^{C}$ can be replaced by $E_{q_{2}}^{C}$ as a generator.

Proposition 4.10 There are elements of $\mathrm{fP}$ and of $\mathcal{R} \mathcal{M}_{2}^{\mathrm{P}}$ that are critical (i.e., non-regular if $\mathrm{P} \neq$ NP), whose product is a non-zero idempotent.

Proof. For $i=0,1$, let $e_{i} \in \mathrm{fP}$ be defined, as a two-variable function, by

$$
e_{i}(w, x)= \begin{cases}\left(w, \phi_{w}(x)\right) & \text { if } x \in i\{0,1\}^{*}, \phi_{w}(x) \in i\{0,1\}^{*}, \text { and }\left|\phi_{w}(x)\right|=|x| \\ \left(w, 0^{|x|}\right) & \text { otherwise. }\end{cases}
$$

Then $\left(e_{1} \circ e_{0}\right)(w, x)=\left(w, 0^{|x|}\right)$ for all $(w, x)$, so $e_{1} \circ e_{0}$ is an idempotent. 
To prove that $e_{i}$ is critical we reduce the satisfiability problem to the inversion problem of $e_{i}$. The reduction for $e_{i}$ maps a boolean formula $B$ with $n$ variables to $\left(b, i^{n} 1\right)$, where $b$ is a program such that $f_{b}(i \tau)=i^{n} B(\tau)$; i.e., for a truth-value assignment $\tau \in\{0,1\}^{n}, f_{b}$ evaluates $B$ on $\tau$, and outputs the resulting truth-value, prefixed with $n$ copies of $i$. If $e_{i}$ were regular then $\operatorname{Im}\left(e_{i}\right)$ would be in $\mathrm{P}$, by Prop. 1.9. Then satisfiability of $B$ could be checked by a P-algorithm, since $B$ is satisfiable iff $\left(b, i^{n} 1\right) \in \operatorname{Im}\left(e_{i}\right)$. To obtain one-variable functions we can take $e_{i}^{C}$.

To prove the proposition for $\mathcal{R M}_{2}^{\mathrm{P}}$ we define $e_{i} \in \mathcal{R} \mathcal{M}_{2}^{\mathrm{P}}$ for $i=0,1$ as follows, first as two-variable functions:

$$
e_{i}(w, x)= \begin{cases}\left(w, \phi_{w}(x)\right) & \text { if } x \in 0 i\{0,1\}^{*}, \phi_{w}(x) \in 0 i\{0,1\}^{*}, \text { and }\left|\phi_{w}(x)\right|=|x| \\ (w, x) & \text { if } x \in 1\{0,1\}^{*} ; \\ \text { undefined } & \text { otherwise. }\end{cases}
$$

Then $\left(e_{1} \circ e_{0}\right)(w, x)=(w, x)$ when $x \in 1\{0,1\}^{*}$, and $\left(e_{1} \circ e_{0}\right)(w, x)$ is undefined otherwise; so $e_{1} \circ e_{0}$ is a partial identity. The reduction of the satisfiability problem to the inversion problem of $e_{i}$ is similar to the case of $\mathrm{fP}$.

\section{$5 \quad$ Reductions and completeness}

The usual reduction between partial functions $f_{1}, f_{2}: A^{*} \rightarrow A^{*}$ is as follows.

Definition $5.1 \quad f_{1}$ is simulated by $f_{2}$ (denoted by $f_{1} \preccurlyeq f_{2}$ ) iff there exist polynomial-time computable partial functions $\beta, \alpha$ such that $f_{1}=\beta \circ f_{2} \circ \alpha$.

Recall polynomial-time many-to-one reduction that is used for languages; it is defined by $L_{1} \preccurlyeq_{\mathrm{m}: 1} L_{2}$ iff for some polynomial-time computable function $\alpha$ and for all $x \in A^{*}: x \in L_{1}$ iff $\alpha(x) \in L_{2}$. This is equivalent to $L_{1}=\alpha^{-1}\left(L_{2}\right)$, and also to $\chi_{L_{1}}=\chi_{L_{2}} \circ \alpha$ (where $\chi_{L_{j}}$ denotes the characteristic function of $L_{j}$ ). So $L_{1} \preccurlyeq \mathrm{m:1} L_{2}$ implies that $\chi_{L_{1}}$ is simulated by $\chi_{L_{2}}$.

Moreover, when we talk about simulations between functions we will always use the following

Addendum to Definition 5.1. We assume that $\beta, \alpha \in \mathrm{fP}$. For a simulation between two right-ideal morphisms of $A^{*}$ we assume $\beta, \alpha \in \mathcal{R} \mathcal{M}_{|A|}^{\mathrm{P}}$.

We can define simulation for monoids in general. For monoids $M_{0} \leq M_{1}$ and $s, t \in M_{1}$, simulation $s \preccurlyeq t$ is the same thing as $s \leq_{\mathcal{J}\left(M_{0}\right)} t$, i.e., the submonoid $\mathcal{J}$-order on $M_{1}$, using multipliers in the submonoid $M_{0}$.

Simulation tells us which functions are harder to compute than others, but it does not say anything about the hardness of inverses of functions. We want a reduction with the property that if a one-way function $f_{1}$ reduces to a function $f_{2} \in \mathrm{fP}$ then $f_{2}$ is also one-way. The intuitive idea is that $f_{1}$ "reduces inversively" to $f_{2}$ iff (1) $f_{1}$ is simulated by $f_{2}$, and (2) the "easiest inverses" of $f_{1}$ are simulated by the "easiest inverses" of $f_{2}$. But "easiest inverses" are difficult to define. We rigorously define inversive reduction as follows.

Definition 5.2 (inversive reduction). Let $f_{1}, f_{2}: A^{*} \rightarrow A^{*}$ be any partial functions. We say that $f_{1}$ reduces inversively to $f_{2}$ (notation, $f_{1} \leqslant$ inv $f_{2}$ ) iff

(1) $f_{1} \preccurlyeq f_{2}$ and

(2) for every inverse $f_{2}^{\prime}$ of $f_{2}$ there exists an inverse $f_{1}^{\prime}$ of $f_{1}$ such that $f_{1}^{\prime} \preccurlyeq f_{2}^{\prime}$.

Here, $f_{1}, f_{2}, f_{1}^{\prime}, f_{2}^{\prime}$ range over all partial functions $A^{*} \rightarrow A^{*}$.

The relation $\leqslant_{\text {inv }}$ can be defined on monoids $M_{0} \leq M_{1} \leq M_{2}$ in general: We let $f_{1}, f_{2}$ range over $M_{1}$, and let inverses $f_{1}^{\prime}, f_{2}^{\prime}$ range over $M_{2}$. For simulation $\preccurlyeq$ we pick $\leq_{\mathcal{J}\left(M_{0}\right)}$ (i.e., multipliers are in $M_{0}$ ). We should assume that $M_{1}$ is regular within $M_{2}$ in order to avoid empty ranges for the quantifiers " $\left(\forall f_{2}^{\prime}\right)\left(\exists f_{1}^{\prime}\right)$ "; otherwise, when $f_{2}$ has no inverse in $M_{2}, f_{1} \leqslant$ inv $f_{2}$ is trivially equivalent to $f_{1} \preccurlyeq f_{2}$. 
Proposition $5.3 \leqslant$ inv is transitive and reflexive.

Proof. Simulation is obviously transitive. Moreover, if $f_{1} \leqslant \operatorname{inv} f_{2}$ and $f_{2} \leqslant \operatorname{inv} f_{3}$, then for each $f_{3}^{\prime}$ there exists an inverse $f_{2}^{\prime}=\beta_{23} \circ f_{3}^{\prime} \circ \alpha_{23}$, and for $f_{2}^{\prime}$ there is an inverse $f_{1}^{\prime}=\beta_{12} \circ f_{2}^{\prime} \circ \alpha_{12}$. Then $f_{1}^{\prime}=\beta_{12} \circ \beta_{23} \circ f_{3}^{\prime} \circ \alpha_{23} \circ \alpha_{12}$, so $f_{3}^{\prime}$ simulates some inverse of $f_{1}$.

Proposition 5.4 If $f_{1} \leqslant \operatorname{inv} f_{2}, f_{2} \in \mathrm{fP}$, and $f_{2}$ is regular, then $f_{1} \in \mathrm{fP}$ and $f_{1}$ is regular. Contrapositive: If $f_{1}, f_{2} \in \mathrm{fP}, f_{1} \leqslant \operatorname{inv} f_{2}$, and $f_{1}$ is one-way, then $f_{2}$ is one-way.

Proof. The property $f_{1} \in \mathrm{fP}$ follows from simulation. If $f_{2}$ is regular, then it has an inverse $f_{2}^{\prime} \in \mathrm{fP}$, and $f_{1}$ has an inverse $f_{1}^{\prime}=\beta \circ f_{2}^{\prime} \circ \alpha$. All the factors are in $\mathrm{fP}$, so $f_{1}^{\prime} \in \mathrm{fP}$.

Definition 5.5 A partial function $g$ is complete (or fP-complete) with respect to $\leqslant$ inv iff $g \in \mathrm{fP}$, and for every $f \in \mathrm{fP}$ we have $f \leqslant$ inv $g$. In a similar way we can define $\mathcal{R} \mathcal{M}_{2}^{\mathrm{P}}$-complete.

Observation: If $g$ is fP-complete then $g \equiv \mathcal{J}$ id $_{A^{*}}$.

Proposition 5.6 The map $\mathrm{ev}_{q_{2}}^{C}$ is $\mathrm{fP}$-complete with respect to inversive reduction.

Proof. Any $\phi_{w} \in \mathrm{fP}$ with $q_{2}$-polynomial program $w$ is simulated by $\operatorname{ev}_{q_{2}}^{C}$; recall formula $(\star)$ in the proof of Prop. 4.5:

$$
\phi_{w}=\pi_{2\left|w^{\prime}\right|+2}^{\prime} \circ \text { contr } \circ \text { recontr }{ }^{2 m} \circ \mathrm{ev}_{q_{2}}^{C} \circ \operatorname{reexpand}^{2 m} \circ \text { expand } \circ \pi_{\text {code }(w) 11},
$$

where $w^{\prime}=\mathrm{co}^{2 m+1} \circ \mathrm{ex}^{2 m+1}(w)$.

To prove the inversive property, let $\mathrm{e}^{\prime}$ be any inverse of $\mathrm{ev}_{q_{2}}^{C}$. We apply $\mathrm{e}^{\prime}$ to a string of the form code $\left(\mathrm{ex}^{2 m+1}(w)\right) 110^{N_{2 m+1}} 11 y$, where $\phi_{\mathrm{ex}^{2 m+1}(w)} \in \mathrm{fP}^{q_{2}}$ and $y \in \operatorname{Im}\left(\phi_{w}\right)$. Thus, $N_{2 m+1}$ is at least as large as the time of the computation that led to output $y$. Note that we use $2 m$ in $N_{2 m+1}$ because the input that led to output $y$ could be polynomially longer than $y$ (by polynomial $q_{2}$ ). Then we have:

$$
\mathrm{e}^{\prime}\left(\operatorname{code}\left(\mathrm{ex}^{2 m+1}(w)\right) 110^{N_{2 m+1}} 11 y\right)=\operatorname{code}\left(\mathrm{ex}^{2 m+1}(w)\right) 110^{N_{2 m+1}} 11 x_{i} \text {, for some } x_{i} \in \phi_{w}^{-1}(y) \text {. }
$$

We don't care whether and how $\mathrm{e}^{\prime}(Z)$ is defined when the input $Z$ is not of the above form. Then $\mathrm{e}^{\prime}$ simulates an inverse $f^{\prime}$ of $\phi_{w}$ defined by

$$
f^{\prime}(y)=\pi_{2\left|w^{\prime}\right|+2}^{\prime} \circ \text { contr } \circ \text { recontr }^{2 m} \circ \mathrm{e}^{\prime} \circ \text { reexpand }^{2 m} \circ \text { expand } \circ \pi_{\text {code }(w) 11}(y)
$$

for all $y \in \operatorname{Im}\left(\phi_{w}\right)$. Indeed, $f^{\prime}(y)=x_{i}\left(\in \phi_{w}^{-1}(y)\right.$ as above $)$.

When $y \notin \operatorname{Im}\left(\phi_{w}\right)$ the right side of the above formula may give a value to $f^{\prime}(y)$; but it does not matter whether and how $f^{\prime}$ is defined outside of $\operatorname{Im}\left(\phi_{w}\right)$. Thus, every inverse of $\mathrm{ev}_{q_{2}}^{C}$ simulates an inverse of $\phi_{w}$.

In a similar way one can prove that the generator-based evaluation map $\mathrm{ev}_{\Gamma, q}$ (for a large enough polynomial $q$ ) is complete in $\mathrm{fP}$.

Notation: For a partial function $f: A^{*} \rightarrow A^{*}$, the set of all inverses $f^{\prime}: A^{*} \rightarrow A^{*}$ of $f$ is denoted by $\operatorname{lnv}(f)$.

Definition 5.7 (uniform inversive reduction). Let $f, g$ be partial functions. An inversive reduction $f \leqslant \operatorname{inv} g$ is called uniform iff $f \preccurlyeq g$, and $(\exists \beta, \alpha \in \mathrm{fP})\left(\forall g^{\prime} \in \operatorname{Inv}(g)\right)\left(\exists f^{\prime} \in \operatorname{Inv}(f)\right)\left[f^{\prime}=\beta \circ g^{\prime} \circ \alpha\right]$. So $\beta$ and $\alpha$ only depend on $f$ and $g$, but not on $g^{\prime}$ or $f^{\prime}$.

We observe that in the proof of Prop. 5.6 the simulation of $f^{\prime}$ by $\mathrm{e}^{\prime}$ only depends on $\phi_{w}$ and e, but not on $f^{\prime}$ nor on $e^{\prime}$. We conclude:

Corollary 5.8 The map $\mathrm{ev}_{q_{2}}^{C}$ is $\mathrm{fP}$-complete with respect to uniform inversive reduction. 
Next we study the completeness of the circuit evaluation map ev $\mathrm{v}_{\text {circ }}$ (defined at the beginning of Section 4). Since it is defined in terms of length-preserving circuits, $\mathrm{ev}_{\text {circ }}$ is itself length-preserving, i.e., it belongs to the submonoid of length-preserving partial functions in $\mathrm{fP}$,

$$
\mathrm{fP}_{\mathrm{Ip}}=\{f \in \mathrm{fP}:|f(x)|=|x| \text { for all } x \in \operatorname{Dom}(f)\} .
$$

Proposition 5.9 The critical map ev $\mathrm{ev}_{\text {circ }}$ is complete in the submonoid $\mathrm{fP}_{\mathrm{lp}}$ with respect to inversive reduction.

Proof. Let $f \in \mathrm{fP}_{\mathrm{Ip}}$ be a fixed length-preserving partial function, and let $M$ be a fixed deterministic polynomial-time Turing machine that computes $f$.

Simulation of $f$ by $\mathrm{ev}_{\text {circ }}$ : It is well known that for every input length $n$ (of inputs of $f$ ) one can construct an acyclic circuit $C_{n}$ such that $C_{n}(x)=f(x)$ for all $x$ of length $n$. The circuit can be constructed from $M$ and $n$ in polynomial time (as a function of $n$ ). Let $\alpha(x)=\left(C_{|x|}, x\right)$, and let $\beta\left(C_{n}, y\right)=y$, where $|y|=n$. Then $f=\beta \circ \mathrm{ev}_{\text {circ }} \circ \alpha$.

Simulation between inverses: Any inverse $e^{\prime}$ of $\mathrm{ev}_{\text {circ }}$ has the form $e^{\prime}(C, y)=\left(C, x_{i}\right)$ for some $x_{i} \in C^{-1}(y)$, when $y \in \operatorname{Im}(C)$. When $y \notin \operatorname{Im}(C), e^{\prime}(C, y)$ could be any pair of bitstrings. Then an inverse $f^{\prime}$ of $f$ is obtained by defining $f^{\prime}(y)=\beta \circ e^{\prime} \circ \alpha(y)$, where $\alpha, \beta$ are as in the simulation of $f$ (in the first part of this proof). Indeed, when $y \in \operatorname{Im}(f)$ we have $\alpha: y \longmapsto\left(C_{n}, y\right)$, where $|y|=n$. Next, $e^{\prime}:\left(C_{n}, y\right) \longmapsto\left(C_{n}, x_{i}\right)$ for some $x_{i} \in C_{n}^{-1}(y)=f^{-1}(y)$; recall that we only use length-preserving circuits, so $|y|=n=\left|x_{i}\right|$. Finally, $\beta:\left(C_{n}, x_{i}\right) \longmapsto x_{i} \in f^{-1}(y)$. So $f^{\prime}$ is an inverse of $f$ on $\operatorname{Im}(f)$; outside of $\operatorname{Im}(f)$, the values of $f^{\prime}$ do not matter.

To show completeness of $\mathrm{ev}_{\text {circ }}$ in $\mathrm{fP}$ (rather than just in $\mathrm{fP}_{\mathrm{Ip}}$ ), a stronger inversive reduction is needed, that overcomes the limitation of length-preservation in $\mathrm{ev}_{\text {circ }}$.

Remark: Circuits are usually generalized to allow the output length to be different from the input length. But that would not simplify the completeness proof for $\mathrm{ev}_{\text {circ }}$, because the main limitation is that all inputs of a circuit have the same length, and all outputs of a circuit have the same length.

Definition 5.10 (polynomial-time Turing simulation). Let $f_{1}, f_{2}: A^{*} \rightarrow A^{*}$ be two partial functions. By definition, $f_{1} \preccurlyeq \mathrm{T} f_{2}$ iff $f_{1}$ is computed by a deterministic polynomial-time Turing machine that can make oracle calls to $f_{2}$; these can include, in particular, calls on the membership problem of $\operatorname{Dom}\left(f_{2}\right)$.

In the next proofs we do not need the full power of Turing reductions. The following, much weaker reduction, will be sufficient.

Notation: Let $L \subseteq A^{*}$. Then $\mathrm{fP}^{L}$ denotes the set of all polynomially balanced partial functions computed by deterministic polynomial-time Turing machines that can make oracle calls to the membership problem of $L$. In particular we will consider $\operatorname{fP}^{\operatorname{Dom}(f)}$ for any partial function $f: A^{*} \rightarrow A^{*}$.

Definition 5.11 (weak Turing simulation). A weak Turing simulation of $f_{1}$ by $f_{2}$ consists of two partial functions $\beta, \alpha$ such that $f_{1}=\beta \circ f_{2} \circ \alpha$, where $\alpha \in \mathrm{fP}^{\operatorname{Dom}\left(f_{2}\right)}$ and $\beta \in \mathrm{fP}$. The existence of a weak Turing simulation of $f_{1}$ by $f_{2}$ is denoted by $f_{1} \preccurlyeq_{\mathrm{wT}} f_{2}$.

Informally we also write $f_{1}=\beta \circ f_{2} \circ \alpha^{\operatorname{Dom}\left(f_{2}\right)}$. In a weak Turing simulation by $f_{2}$, only the domain of $f_{2}$ is repeatedly queried; $f_{2}$ itself is called only once, and this call of $f_{2}$ takes the form of an ordinary (not a Turing) simulation.

Definition 5.12 (inversification of a simulation). For any simulation relation $\preccurlyeq x$ between partial functions, the corresponding inversive reduction $\leqslant \mathrm{inv}, \mathrm{x}$ is defined as follows:

$f_{1} \leqslant \mathrm{inv}, \mathrm{x} f_{2} \quad$ iff

$f_{1} \preccurlyeq x f_{2}$, and for every inverse $f_{2}^{\prime}$ of $f_{2}$ there exists an inverse $f_{1}^{\prime}$ of $f_{1}$ such that $f_{1}^{\prime} \preccurlyeq x f_{2}^{\prime}$. 
One easily proves:

Proposition 5.13 If $\preccurlyeq \mathrm{x}$ is transitive then $\leqslant_{\mathrm{inv}, \mathrm{x}}$ is transitive.

Based on this general definition we can introduce polynomial-time inversive Turing reductions, denoted by $\leqslant_{\text {inv, } \mathrm{T}}$, and polynomial-time inversive weak Turing reductions, denoted by $\leqslant_{\text {inv, } \mathrm{wT}}$. The following is straightforward to prove.

Proposition 5.14 If $f_{1} \leqslant \mathrm{inv}, \mathrm{T} f_{2}$ then:

- $f_{2} \in \mathrm{fP}$ implies $f_{1} \in \mathrm{fP}$;

- $f_{2} \in \mathrm{fP}$ and $f_{2}$ is regular, implies $f_{1}$ is regular.

The following shows that $\leqslant$ inv,wT can overcome the limitations of length-preservation.

Proposition 5.15 For every $f \in \mathrm{fP}$ there exists $\ell \in \mathrm{fP}_{\mathrm{Ip}}$ such that $f \leqslant_{\mathrm{inv}, \mathrm{w} T} \ell$.

Proof. For any $f \in \mathrm{fP}$ we define $\ell_{f} \in \mathrm{fP}_{\mathrm{Ip}}$ by

$$
\ell_{f}\left(0^{n} 1 x\right)= \begin{cases}0^{|x|} 1 f(x) & \text { if } n=|f(x)|, \\ \text { undefined } & \text { on all other inputs. }\end{cases}
$$

Let $p_{f}($.$) be a polynomial upper-bound on the time-complexity and on the balance of f$.

1. Proof that $f \preccurlyeq \ell_{f}$ (simulation): We have $f=\beta \circ \ell_{f} \circ \alpha$, where $\alpha(x)=0^{|f(x)|} 1 x$ for all $x \in A^{*}$; and $\beta\left(0^{m} 1 y\right)=y$ for all $y \in A^{*}$ and all $m \leq p_{f}(|y|) \quad(\beta$ is undefined otherwise).

2. Proof that for every inverse $\ell^{\prime}$ of $\ell_{f}$ there is an inverse $f^{\prime}$ of $f$ such that $f^{\prime} \preccurlyeq_{w T} \ell_{f}^{\prime}$ :

Every element of $\operatorname{Im}\left(\ell_{f}\right)$ has the form $0^{m} 1 y$ where $y \in \operatorname{Im}(f)$, for some appropriate $m$. More precisely, $\ell_{f}^{-1}\left(0^{m} 1 y\right)=\left\{0^{|y|} 1 x: x \in f^{-1}(y) \cap A^{m}\right\}$. Hence, $0^{m} 1 y \in \operatorname{Im}\left(\ell_{f}\right)$ iff $f^{-1}(y) \cap A^{m} \neq \varnothing$. Therefore, any inverse $\ell^{\prime}$ satisfies $\ell^{\prime}\left(0^{m} 1 y\right)=0^{|y|} 1 x_{i}$ for some choice of $x_{i} \in f^{-1}(y) \cap A^{m}$; we do not care about the values of $\ell^{\prime}$ when its inputs are not in $\operatorname{Im}\left(\ell_{f}\right)$. Thus we can define an inverse of $f$ on each $y \in \operatorname{Im}(f)$ by

$$
f^{\prime}(y)=x_{i}, \text { for } x_{i} \text { chosen in } f^{-1}(y) \cap A^{m}
$$

where $m$ is the minimum integer such that $f^{-1}(y) \cap A^{m} \neq \varnothing$.

We don't care what $f^{\prime}(y)$ is when $y \notin \operatorname{Im}(f)$.

To obtain an inversive weak Turing reduction we need to compute $x_{i}=f^{\prime}(y)$ from $y$, based on oracle calls to $\operatorname{Dom}\left(\ell^{\prime}\right)$ and one simulation of $\ell^{\prime}$. This computation of $x_{i}$ is done in two steps: First we compute the minimum $m\left(=\left|x_{i}\right|\right)$ such that $f^{-1}(y) \cap A^{m} \neq \varnothing$ (see Step 1 below for details). Second, we apply $\ell^{\prime}$ to compute $\ell^{\prime}\left(0^{\left|x_{i}\right|} 1 y\right)=0^{|y|} 1 x_{i}$. From this we obtain $x_{i}$ by applying the map $\beta$ defined above (in part 1 of this proof). The first step is a Turing reduction to the domain of $\ell^{\prime}$. The second step is a simulation by $\ell^{\prime}$. In more detail:

Step 1: By input balance we have $\left|x_{i}\right| \leq p_{f}(|y|)$ when $x_{i} \in f^{-1}(y)$. For each $m \in\left\{0,1, \ldots, p_{f}(|y|)\right\}$, in increasing order, we make an oracle call to the membership problem in $\operatorname{Dom}\left(\ell^{\prime}\right)$ with query input $0^{m} 1 y$. If $y \in \operatorname{Im}(f)$ then the first of these queries with a positive answer determines $m$, and $0^{m} 1 y$ is returned.

Step 2: To the result $0^{m} 1 y$ of step 1 we apply the functions $\ell^{\prime}$ and $\beta$. This yields $x_{i}$, which is $f^{\prime}(y)$. Thus, step 2 is just a simulation.

Togetherm, steps 1 and 2 form a weak polynomial Turing simulation of $f^{\prime}$ by $\ell^{\prime}$.

Corollary 5.16 The critical map ev $\mathrm{v}_{\text {circ }}$ is $\mathrm{fP}$-complete with respect to composites of polynomial inversive weak Turing reductions and polynomial inversive simulation reductions ( $\leqslant$ inv,wT and $\leqslant_{\text {inv }}$ ). 
Proof. For every $f \in \mathrm{fP}$ we first reduce $f$ to a length-preserving function $\ell_{f}$, by Prop. 5.15, Then we reduce $\ell_{f}$ to ev $v_{\text {circ }}$ by Prop. 5.9.

\section{Reduction and completeness in $\mathcal{R} \mathcal{M}_{2}^{\mathrm{P}}$}

The following shows that the encoding that embeds fP into $\mathcal{R} \mathcal{M}_{2}^{\mathrm{P}}$ does not make inversion easier.

Proposition 5.17 For all $f \in \mathrm{fP}$ we have $f \leqslant_{\mathrm{inv}} f^{C}$, where $\leqslant_{\mathrm{inv}}$ is based on simulation in $\mathrm{fP}$.

Proof. Recall the encoding maps $(.)_{\#}: x \in\{0,1\}^{*} \longmapsto x \# \in\{0,1, \#\}^{*}$, and code which replaces 0,1 , \# by respectively 00,01,11, defined in Section 3; and recall $f^{C}$ from Def. 3.2. We now introduce inverses of these maps. Let $\operatorname{dec}: \operatorname{code}(x) \in\{00,01,11\}^{*} \longmapsto x \in\{0,1\}^{*}$ (undefined outside of $\{00,01,11\}^{*}$ ), and $r: x \# \longmapsto x \in\{0,1\}^{*}$ (undefined outside of $\{0,1\}^{*} \#$ ). Then

$$
f=r \circ \operatorname{dec} \circ f^{C} \circ \operatorname{code} \circ(.)_{\#} .
$$

Clearly, (.) $)_{\#}$, code, dec, $r \in \mathrm{fP}$. Hence $f^{C}$ simulates $f$.

For the inversive part of the reduction, let $\varphi^{\prime}$ be any inverse of $f^{C}$; we want to find an inverse $f^{\prime}$ of $f$ such that $f^{\prime} \preccurlyeq \varphi^{\prime}$, where $\preccurlyeq$ denotes simulation in fP. Any element of $\operatorname{Im}\left(f^{C}\right)$ has the form code $(s) 11 t$, with $s, t \in\{0,1\}^{*}$, and $s \in \operatorname{Im}(f)$. Moreover, if code $(s) 11 t \in \operatorname{Im}\left(f^{C}\right)$ then $\operatorname{code}(s) 11 \in \operatorname{Im}\left(f^{C}\right)$. Let us define $f^{\prime}$ for any $s \in \operatorname{Im}(f)$ by $f^{\prime}(s)=x_{1}$ where $x_{1}$ is such that $\varphi^{\prime}(\operatorname{code}(s) 11)=\operatorname{code}\left(x_{1}\right) 11$ $\in\left(f^{C}\right)^{-1}(\operatorname{code}(s) 11)$. Then $x_{1} \in f^{-1}(s)$. In general, finally, we define $f^{\prime}$ by

$$
f^{\prime}=r \circ \operatorname{dec} \circ \varphi^{\prime} \circ \text { code } \circ(.)_{\#} \text {. }
$$

For $s \in \operatorname{Im}(f)$ we indeed have then: $r \circ \operatorname{dec} \circ \varphi^{\prime} \circ$ code $\circ(.)_{\#}(s)=r \circ \operatorname{dec} \circ \varphi^{\prime}(\operatorname{code}(s) 11)=$ $r \circ \operatorname{dec}\left(\operatorname{code}\left(x_{1}\right) 11\right)=x_{1}$, where $x_{1} \in f^{-1}(s)$, as above. So this definition makes $f^{\prime}$ an inverse of $f$ on $\operatorname{Im}(f)$; hence $f^{\prime}$ is an inverse of $f$. The above formula for $f^{\prime}$ explicitly shows that $f^{\prime} \preccurlyeq \varphi^{\prime}$.

Let $\equiv_{\text {inv }}$ denote $\leqslant_{\text {inv-equivalence }}$ (i.e., $f \equiv_{\text {inv }} g$ iff $f \leqslant_{\text {inv }} g$ and $g \leqslant_{\text {inv }} f$ ). The $\leqslant_{\text {inv }}$-complete functions of fP obviously form an $\equiv_{i n v}$-class, and this is the maximum class for the $\leqslant_{\text {inv }}$-preorder. Similarly, the complete functions of $\mathcal{R} \mathcal{M}_{2}^{\mathrm{P}}$ are the maximum inversive reducibility class in $\mathcal{R} \mathcal{M}_{2}^{\mathrm{P}}$. The non-empty regular elements of $\mathcal{R} \mathcal{M}_{2}^{\mathrm{P}}$ also form an equivalence class in $\mathcal{R} \mathcal{M}_{2}^{\mathrm{P}}$, and this is the minimum class of all non-empty functions, as the following shows:

Proposition 5.18 For every $f, r \in \mathcal{R} \mathcal{M}_{2}^{\mathrm{P}}$ where $r$ is regular and $f$ is non-empty, we have $r \leqslant$ inv $f$.

Proof. The simulation $r \preccurlyeq f$ follows from $\mathcal{J}^{0}$-simplicity of $\mathcal{R} \mathcal{M}_{2}^{\mathrm{P}}$. Let $f^{\prime}$ be any inverse of $f$ (with $f^{\prime}$ not necessarily in $\left.\mathcal{R} \mathcal{M}_{2}^{\mathrm{P}}\right)$. Since $r$ is regular, there is an inverse $r^{\prime} \in \mathcal{R} \mathcal{M}_{2}^{\mathrm{P}}$ of $r$. Since $f^{\prime}$ is not the empty map there exist $x_{0}, y_{0}$ with $f^{\prime}\left(y_{0}\right)=x_{0}$. Then $\left(x_{0} \leftarrow y_{0}\right)$ is simulated by $f^{\prime}$, since $\left(x_{0} \leftarrow y_{0}\right)=\mathrm{id}_{\left\{x_{0}\right\}} \circ f^{\prime}$. Moreover, $\left(x_{0} \leftarrow y_{0}\right)$ is regular and $\left(x_{0} \leftarrow y_{0}\right)$ belongs to $\mathcal{R} \mathcal{M}_{2}^{\mathrm{P}}$, so $\left(x_{0} \leftarrow y_{0}\right)$ simulates $r^{\prime}$ (again by $\mathcal{J}^{0}$-simplicity of $\mathcal{R} \mathcal{M}_{2}^{\mathrm{P}}$ ). Thus, $f^{\prime}$ simulates $r^{\prime}$.

Proposition 5.19 In both $\mathrm{fP}$ and $\mathcal{R} \mathcal{M}_{2}^{\mathrm{P}}$ : The $\equiv_{\mathcal{D}}$-relation is a refinement of $\equiv_{\mathrm{inv}}$.

Proof. Is suffices to prove that both $\equiv_{\mathcal{R}}$ and $\equiv_{\mathcal{L}}$ refine $\equiv_{\text {inv }}$. We will prove that $f \equiv_{\mathcal{R}} g$ implies $f \equiv_{\text {inv }} g$. (The same reasoning works for $\equiv_{\mathcal{L}}$.) When $f \equiv_{\mathcal{R}} g$, there exist $\alpha, \beta \in \mathrm{fP}\left(\right.$ or $\left.\in \mathcal{R} \mathcal{M}_{2}^{\mathrm{P}}\right)$ such that $f=g \alpha$ and $g=f \beta$. So, $f$ and $g$ simulate each other.

For any inverse $f^{\prime}$ of $f$ we have $f=f f^{\prime} f=g \alpha f^{\prime} f$. Right-multiplying by $\beta$ we obtain $g=g \alpha f^{\prime} g$, hence $\alpha f^{\prime}$ is an inverse of $g$, and $\alpha f^{\prime}$ is obviously simulated by $f^{\prime}$. So, $g$ inversely reduces to $f$. Similarly, $f$ inversely reduces to $g$. 


\section{The polynomial hierarchy}

The classical polynomial hierarchy for languages is defined by $\Sigma_{1}^{\mathrm{P}}=\mathrm{NP}, \Pi_{1}^{\mathrm{P}}=$ coNP, and for all $k>0$ : $\Sigma_{k+1}^{\mathrm{P}}=\mathrm{NP}^{\Sigma_{k}^{\mathrm{P}}}$ (i.e., all languages accepted by nondeterministic Turing machines with oracle in $\Sigma_{k}^{\mathrm{P}}$, or equivalently, with oracle in $\left.\Pi_{k}^{\mathrm{P}}\right)$; and $\Pi_{k+1}^{\mathrm{P}}=(\operatorname{coNP})^{\Sigma_{k}^{\mathrm{P}}}\left(=\operatorname{co}\left(\mathrm{NP}^{\Sigma_{k}^{\mathrm{P}}}\right)\right)$. Moreover, $\mathrm{PH}=\bigcup_{k} \Sigma_{k}^{\mathrm{P}}$ ( $\subseteq$ PSpace).

Polynomial hierarchy for functions:

$\mathrm{fP}^{\Sigma_{k}^{\mathrm{P}}}$ consists of all polynomially balanced partial functions $A^{*} \rightarrow A^{*}$ computed by deterministic polynomial-time Turing machines with oracle in $\Sigma_{k}^{\mathrm{P}}$ (or equivalently, with oracle in $\Pi_{k}^{\mathrm{P}}$ );

$\mathrm{fP}^{\mathrm{PH}}$ consists of all polynomially balanced partial functions $A^{*} \rightarrow A^{*}$ computed by deterministic polynomial-time Turing machines with oracle in $\mathrm{PH}$. Equivalently, $\mathrm{fP}^{\mathrm{PH}}=\bigcup_{k} \mathrm{fP}^{\Sigma_{k}^{\mathrm{P}}}$.

Moreover, fPSpace consists of all polynomially balanced partial functions (on $A^{*}$ ) computed by deterministic polynomial-space Turing machines.

We can also define a polynomial hierarchy over $\mathcal{R} \mathcal{M}_{2}^{\mathrm{P}}$.

Proposition 6.1 Every $f \in \mathrm{fP}$ has an inverse in $\mathrm{fP}^{\Sigma_{1}^{\mathrm{P}}}$, and every $f \in \mathrm{fP}^{\Sigma_{k}^{\mathrm{P}}}$ has an inverse in $\mathrm{fP}^{\Sigma_{k+1}^{\mathrm{P}}}$. The monoids $\mathrm{fP}^{\mathrm{PH}}$ and $\mathrm{fPSpace}$ are regular.

Proof. The following is an inverse of $f$ :

$$
f_{\min }^{\prime}(y)= \begin{cases}\min \left(f^{-1}(y)\right) & \text { if } y \in \operatorname{Im}(f), \\ \text { undefined } & \text { otherwise }\end{cases}
$$

where $\min (S)$ denotes the minimum of a set of strings $S$ in dictionary order (or alternatively in lengthlexicographic order). To show that $f_{\min }^{\prime} \in \mathrm{fP}^{\mathrm{NP}}$ when $f \in \mathrm{fP}$ we first observe that for any fixed $f \in \mathrm{fP}$ the following problems are in NP:

(1) On input $y \in A^{*}$, decide whether $y \in \operatorname{Im}(f)$.

(2) Fix $u \in A^{*}$; on input $y \in A^{*}$, decide whether $y \in f\left(u A^{*}\right)$ (i.e., decide whether there exists $x \in u A^{*}$ such that $f(x)=y)$.

When $y \notin \operatorname{Im}(f)$ then it doesn't matter what value we choose for $f_{\min }^{\prime}(y)$; we choose $f_{\min }^{\prime}(y)$ to be undefined then.

Here is an $\mathrm{fP}^{\mathrm{NP}}$-algorithm for computing $f_{\min }^{\prime}(y)$. It is a form of binary search in the sorted list $A^{*}$, that ends when a string in $f^{-1}(y)$ has been found. A growing prefix $z$ of $x=f_{\min }^{\prime}(y)$ is constructed; at each step we query whether $z$ can be extended by a 0 or a 1 ; i.e., we ask whether $y \in f\left(z 0 A^{*}\right)$; we don't need to ask whether $y \in f\left(z 1 A^{*}\right)$ too, since we tested already that $y \in \operatorname{Im}(f)$. Oracle calls are denoted by angular brackets $\langle\ldots\rangle$, and $\varepsilon$ denotes the empty word.

Algorithm for $f_{\min }^{\prime}$ on input $y$ :

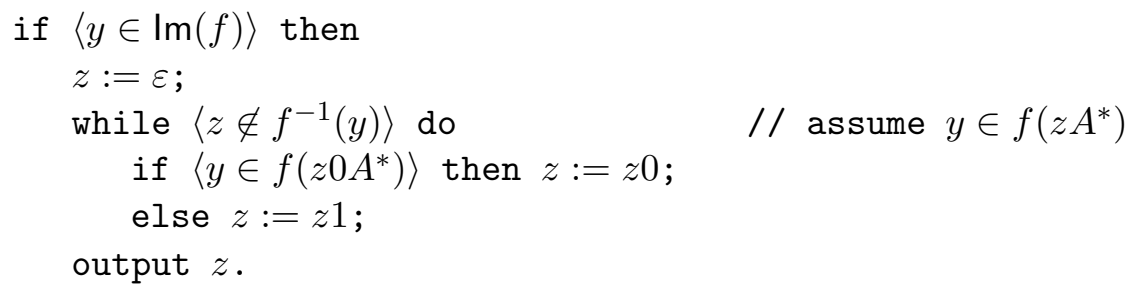

One can prove in a similar way that when $f \in \mathrm{fP}^{\Sigma_{k}^{\mathrm{P}}}$ then $f_{\min }^{\prime} \in \mathrm{fP}^{\Sigma_{k+1}^{\mathrm{P}}}$ : In that case the problems (1) and (2) above are in $\mathrm{NP}^{\Sigma_{k}^{\mathrm{P}}}=\Sigma_{k+1}^{\mathrm{P}}$.

The regularity of $\mathrm{fP}^{\mathrm{PH}}$ follows immediately from the fact about $\mathrm{fP}^{\Sigma_{k}^{\mathrm{P}}}$ for each $k$. The regularity of fPSpace holds because the above algorithm can be carried out in fPSpace.

The above algorithm is similar to the proofs in the literature that $\mathrm{P} \neq \mathrm{NP}$ iff one-way functions exist; see e.g. [15] p. 33. 
In the proof of Prop. 6.1 we used the function $f_{\min }^{\prime}$. In a similar way, by using $\max \left(f^{-1}(y)\right)$ one can define $f_{\max }^{\prime} \in \mathrm{fP}^{\Sigma_{1}^{\mathrm{P}}}$, which is also an inverse of $f$. Yet more inverses can be defined: for any positive integer $i$ let

$$
f_{i}^{\prime}(y)= \begin{cases}i^{\text {th }} \text { word in } f^{-1}(y) & \text { if } y \in \operatorname{Im}(f) \\ \text { undefined } & \text { otherwise. }\end{cases}
$$

Here, " $i$ th word" refers to the dictionary order; also, when $i>\left|f^{-1}(y)\right|$, the $i^{\text {th }}$ word is taken to be the maximum word in $f^{-1}(y)$. Then $f_{i}^{\prime}$ is an inverse of $f$ and $f_{i}^{\prime} \in \mathrm{fP}^{\Sigma_{1}^{\mathrm{P}}}$; note that $i$ is fixed for each function $f_{i}^{\prime}$.

Proposition 6.2 For any fP-critical partial function $f \in \mathrm{fP}$ we have: $f$ is one-way iff $f_{\min }^{\prime} \notin \mathrm{fP}$. Similarly, $f$ is one-way iff $f_{\max }^{\prime} \notin \mathrm{fP} \quad$ iff $(\exists i>0)\left[f_{i}^{\prime} \notin \mathrm{fP}\right]$.

Proof. Since $f_{\min }^{\prime}$ is an inverse of $f$, the direction " $\Rightarrow$ " is obvious by the definition of one-way function. Conversely, we saw that if $f \in \mathrm{fP}$ then $f_{\text {min }}^{\prime} \in \mathrm{fP}^{\Sigma_{1}^{\mathrm{P}}}$. If $f_{\min }^{\prime} \notin \mathrm{fP}$ then $\mathrm{fP} \neq \mathrm{fP}^{\Sigma_{1}^{\mathrm{P}}}$, hence $\mathrm{P} \neq \mathrm{NP}$, hence one-way functions exist. Then any $\mathrm{fP}$-critical function $f$ is one-way.

Recall that a partial function $f$ is called fP-critical iff $f \in \mathrm{fP}$ and the existence of one-way functions implies that $f$ is one-way. In particular, fP-complete functions (with respect to inversive reduction) are fP-critical. An interesting consequence of the above Proposition is that now we do not only have critical functions, but these functions also have critical inverses.

Definition 6.3 Let $f$ be an $\mathrm{fP}$-critical function. We say that an inverse $f^{\prime}$ of $f$ is a critical inverse of $f$ iff $f^{\prime} \notin \mathrm{fP}$ implies that $f$ is one-way.

Corollary 6.4 For the fP-critical function $\mathrm{ev}_{\mathrm{circ}}$, the inverses $\left(\mathrm{ev}_{\mathrm{circ}}\right)_{\min }^{\prime},\left(\mathrm{ev}_{\mathrm{circ}}\right)_{\max }^{\prime}$ and $\left(\mathrm{ev}_{\mathrm{circ}}\right)_{i}^{\prime}$ are critical inverses.

Thus, to decide whether $\mathrm{P} \neq \mathrm{NP}$ it suffices to consider one function, and one of its inverses.

Proposition 6.5 For each $k \geq 1$ the monoid $\mathrm{fP}^{\Sigma_{k}^{\mathrm{P}}}$ is finitely generated, but not finitely presented. The monoid fPSpace is also finitely generated, but not finitely presented.

The monoid $\mathrm{fP}^{\mathrm{PH}}$ is not finitely generated, unless the polynomial hierarchy collapses.

Proof. The proof for fPSpace is similar to the proof that we gave for fP in Prop. 4.8.

For $\mathrm{fP}^{\Sigma_{k}^{\mathrm{P}}}$, let $Q_{k}$ be any $\Sigma_{k}^{\mathrm{P}}$-complete problem; we can assume that all oracle calls are calls to $Q_{k}$. Then every $f \in \mathrm{fP}^{\Sigma_{k}^{\mathrm{P}}}$ has a program which is like an fP-program, but with oracle calls to $Q_{k}$ added. For every polynomial $q \geq q_{2}$, an evaluation function $\mathrm{ev}_{q}^{Q_{k}}$ for $\mathrm{fP}^{\Sigma_{k}^{\mathrm{P}}}$ can then be designed; in the computation of $\operatorname{ev}_{q}^{Q_{k}}(w, x)$, oracle calls to $Q_{k}$ are made whenever the program $w$ being executed makes calls to $Q_{k}$. Then, $\operatorname{ev}_{q}^{Q_{k}}(w, x)=\left(w, \phi_{w}(x)\right)$. By using $\operatorname{ev}_{q}^{Q_{k}}$ the proof for $\mathrm{fP}^{\Sigma_{k}^{\mathrm{P}}}$ is similar to the proof of Prop. 4.8,

If $\mathrm{fP}^{\mathrm{PH}}$ were finitely generated then let $m$ be the lowest level in the hierarchy that contains a finite generating set. Then $\mathrm{fP}^{\mathrm{PH}} \subseteq \mathrm{fP}^{\Sigma_{m}^{\mathrm{P}}}$.

Instead of using all of $\mathrm{fP}^{\mathrm{NP}}$ to obtain inverses for the elements of $\mathrm{fP}$, we could simply adjoin inverses to $\mathrm{fP}$ (within $\mathrm{fP}^{\mathrm{NP}}$ ). It turns out that it suffices to adjoin just one inverse $e^{\prime} \in \mathrm{fP}^{\mathrm{NP}}$ of a function $e$ that is fP-complete for $\leqslant$ inv.

Notation: For a semigroup $S$ and a subset $W \subseteq S$, the subsemigroup of $S$ generated by $W$ is denoted by $\langle W\rangle_{S}$. For any $h \in \mathrm{fP}^{\mathrm{NP}}$, we denote $\langle\mathrm{fP} \cup\{h\}\rangle_{\mathrm{fP}}$ 列 $\mathrm{fP}[h]$ (called "fP with $h$ adjoined"). So, $\quad \mathrm{fP} \subseteq \mathrm{fP}[h] \subseteq \mathrm{fP}^{\mathrm{NP}}$.

Proposition 6.6 Let $g \in \mathrm{fP}$ be any function that is $\mathrm{fP}$-complete with respect to $\leqslant_{\text {inv }}$, and let $g^{\prime}$ be any inverse of $g$ such that $g^{\prime} \in \mathrm{fP}^{\mathrm{NP}}$. Then the subsemigroup $\mathrm{fP}\left[g^{\prime}\right]$ of $\mathrm{fP}^{\mathrm{NP}}$ contains at least one inverse of each element of $\mathrm{fP}$. 
Proof. From the assumption that $g$ is complete it follows that

$$
(\forall f \in \mathrm{fP})\left(\forall g^{\prime} \in \operatorname{lnv}(g) \cap \mathrm{fP}^{\mathrm{NP}}\right)\left(\exists f^{\prime} \in \operatorname{lnv}(f)\right)(\exists \beta, \alpha \in \mathrm{fP})\left[f^{\prime}=\beta g^{\prime} \alpha\right] .
$$

So for any fixed $g^{\prime} \in \operatorname{lnv}(g) \cap \mathrm{fP}^{\mathrm{NP}}$, every $f \in \mathrm{fP}$ has an inverse of the form $f^{\prime}=\beta g^{\prime} \alpha$, for some $\beta, \alpha \in \mathrm{fP}$ (that depend on $f^{\prime}$ ). Hence $f^{\prime} \in \mathrm{fP}\left[g^{\prime}\right]$.

\section{Observations:}

1. We saw in the proof of Prop. 6.5 that $\mathrm{fP}^{\mathrm{NP}}$ has complete elements with respect to simulation. For any $\mathrm{fP}^{\mathrm{NP}}$-complete element $h$ we have $\mathrm{fP}^{\mathrm{NP}}=\mathrm{fP}[h]$. This raises the question: Is $\mathrm{fP}^{\mathrm{NP}} \neq \mathrm{fP}\left[g^{\prime}\right]$, when $g^{\prime} \in \mathrm{fP}^{\mathrm{NP}}$ and $g^{\prime}$ is an inverse of an element $g$ that is $\mathrm{fP}$-complete (for $\leqslant$ inv $)$ ? In one direction we have:

If there exists $g$ which is $\mathrm{fP}$-complete with respect to $\leqslant$ inv, and an inverse $g^{\prime} \in \mathrm{fP}^{\mathrm{NP}}$ such that $\mathrm{fP}^{\mathrm{NP}} \neq \mathrm{fP}\left[g^{\prime}\right]$, then $\mathrm{P} \neq \mathrm{NP}$.

Indeed, if $\mathrm{fP}^{\mathrm{NP}} \neq \mathrm{fP}\left[g^{\prime}\right]$ then $\mathrm{fP} \subseteq \mathrm{fP}\left[g^{\prime}\right] \varsubsetneqq \mathrm{fP}^{\mathrm{NP}}$, hence $\mathrm{fP} \neq \mathrm{fP}^{\mathrm{NP}}$, hence $\mathrm{P} \neq \mathrm{NP}$.

2. If there exist $g_{1}, g_{2}$ (not necessarily different) that are $\mathrm{fP}$-complete with respect to $\leqslant_{\text {inv }}$, and inverses $g_{1}^{\prime}, g_{2}^{\prime} \in \mathrm{fP} N$ of $g_{1}$, respectively $g_{2}$, such that $\mathrm{fP}\left[g_{1}^{\prime}\right] \neq \mathrm{fP}\left[g_{2}^{\prime}\right]$, then $\mathrm{P} \neq \mathrm{NP}$.

Indeed, by contraposition, if $\mathrm{P}=\mathrm{NP}$ then $\mathrm{fP}=\mathrm{fP} \mathrm{NP}$, hence $g_{1}^{\prime}, g_{2}^{\prime} \in \mathrm{fP}$. Then $\mathrm{fP}\left[g_{1}^{\prime}\right]=\mathrm{fP}=\mathrm{fP}\left[g_{2}^{\prime}\right]$.

3. The following two statements are equivalent: (1) $\mathrm{P} \neq \mathrm{NP}$; (2) there exist $g$ which is $\mathrm{fP}$-complete with respect to $\leqslant_{\mathrm{inv}}$, and an inverse $g^{\prime} \in \mathrm{fP}^{\mathrm{NP}}$ such that $\mathrm{fP} \neq \mathrm{fP}\left[g^{\prime}\right]$.

Indeed, if such a $g$ and $g^{\prime}$ exist then $g$ is a one-way function, hence $\mathrm{P} \neq \mathrm{NP}$. If for such a $g$ and $g^{\prime}$ we have $\mathrm{fP}=\mathrm{fP}\left[g^{\prime}\right]$, then $g$ is an $\mathrm{fP}$-complete function which is not one-way, hence one-way functions do not exist.

\section{Other monoids:}

(1) We have: $\mathrm{fP}^{\mathrm{PS} \text { pace }}=\mathrm{fPS}$ pace.

Indeed, the monoid fPPSace consists of polynomially balanced functions that are polynomial-time computable, with calls to PSpace oracles. The monoid fPSpace consists of polynomially balanced functions that are polynomial-space computable (hence they might use exponential time). Obviously, $\mathrm{fP}^{\mathrm{PS} \text { pace }} \subseteq$ fPSpace. But the converse holds too, since the polynomially many output bits of a function in fPSpace can be found one by one, by a polynomial number of calls to PSpace oracles.

(2) We define fLog ("functions in log-space") to consist of the polynomially balanced partial functions that are computable in deterministic log space. fLog is closed under composition (see [16]), and $\mathrm{fLog} \subseteq \mathrm{fP}$.

If $\mathrm{P} \neq \mathrm{NP}$ then $\mathrm{fLog}$ is non-regular; more strongly, in that case fLog contains one-way functions (with no inverse in $\mathrm{fP}$ ). Indeed, the $3 \mathrm{CNF}$ satisfiability problem reduces to the inversion of the map $(B, \alpha) \mapsto(B, B(\alpha))$, where $B$ is any boolean formula in $3 \mathrm{CNF}$, and $\alpha$ is a truth-value assignment for $B$. It is not difficult to prove that this map is in fLog when $B$ is in $3 \mathrm{CNF}$. One of the referees observed that $\mathrm{fLog}$ is regular iff NP $=\mathrm{L}$, i.e., the class of languages accepted in deterministic log-space.

(3) We define fLin ("functions in linear time") to consist of the linearly balanced partial functions that are computable in deterministic linear time. fLin is closed under composition, and it is non-regular iff $\mathrm{P} \neq \mathrm{NP}$. More strongly, if $\mathrm{P} \neq \mathrm{NP}$ then fLin contains one-way functions (with no inverse in $\mathrm{fP}$ ); this is proved by padding arguments.

Acknowledgement: This paper benefitted greatly form corrections offered by the referees.

\section{References}

[1] S. Arora, B. Barak, Computational Complexity, Cambridge U.P. (2009).

[2] J.C. Birget, "One-way permutations, computational asymmetry and distortion", J. of Algebra, Computational Section, 320(11) (2008) 4030-4062. 
[3] J.C. Birget, "Monoid generalizations of the Richard Thompson groups", J. of Pure and Applied Algebra, 213(2) (2009) 264-278.

[4] J.C. Birget, "The $\mathcal{R}$ - and $\mathcal{L}$-orders of the Thompson-Higman monoid $M_{k, 1}$ and their complexity", International J. of Algebra and Computation, 20.4 (2010) 489-524.

[5] J.C. Birget, "Circuits, coNP-completeness, and the groups of Richard Thompson", International J. of Algebra and Computation 16(1) (Feb. 2006) 35-90.

[6] J.C. Birget, "The groups of Richard Thompson and complexity", International J. of Algebra and Computation 14(5,6) (Dec. 2004) 569-626.

[7] J.C. Birget, "On the circuit-size of inverses", International Journal of Foundations of Computer Science, 22.8 (2011) 1925-1938.

[8] J. W. Cannon, W. J. Floyd, W. R. Parry, "Introductory notes on Richard Thompson's groups", L'Enseignement Mathématique 42 (1996) 215-256.

[9] A.H. Clifford, G.B. Preston, The Algebraic Theory of Semigroups, Vol. 1 (Mathematical Survey, No 7 (I)) American Mathematical Society, Providence (1961).

[10] W. Diffie, M. Hellman, "New directions in cryptography", IEEE Transactions on Information Theory 22.6 (1976) 644-654.

[11] D.Z. Du, K.I. Ko, Theory of Computational Complexity, Wiley (2000).

[12] O. Goldreich, Foundations of Cryptography: Basic Tools, Cambridge U.P. (2001).

[13] P.A. Grillet, Semigroups, An Introduction to the Structure Theory, Marcel Dekker, New York (1995).

[14] J. Hartmanis, Feasible Computations and Provable Complexity Properties, CBMS-NSF Regional Conference Series in Applied Mathematics 30, SIAM (1978).

[15] L.H. Hemaspaandra, M. Ogihara, The Complexity Theory Companion, Springer 2002.

[16] J.E. Hopcroft, J.D. Ullman, Introduction to Automata Theory, Languages and Computation, AddisonWesley (1979).

[17] G. Higman, "Finitely presented infinite simple groups", Notes on Pure Mathematics 8, The Australian National University, Canberra (1974).

[18] L. Levin, "One-way functions and pseudorandom generators", Combinatorica 7.4 (1987) 357-363.

[19] L. Levin, "The tale of one-way functions", Problemy Peredatshi Informatsii 39.1 (2003) 92-103.

[20] R. McKenzie, R. J. Thompson, "An elementary construction of unsolvable word problems in group theory", in Word Problems, (W. W. Boone, F. B. Cannonito, R. C. Lyndon, editors), North-Holland (1973) pp. 457-478.

[21] A.J. Menezes, P. van Oorschot, S.A. Vanstone, Handbook of Applied Cryptography, CRC Press (1996).

[22] Ch. Papadimitriou, Computational Complexity, Addison-Wesley (1994).

[23] D. Perrin, J.E. Pin, Infinite Words, Elsevier (2004).

[24] Elizabeth A. Scott, "A construction which can be used to produce finitely presented infinite simple groups", J. of Algebra 90 (1984) 294-322.

[25] Richard J. Thompson, "Embeddings into finitely generated simple groups which preserve the word problem", in Word Problems II, (S. Adian, W. Boone, G. Higman, editors), North-Holland (1980) pp. 401-441.

J.C. Birget

Dept. of Computer Science

Rutgers University - Camden

Camden, New Jersey

birget@camden.rutgers.edu 